TRANSACTIONS OF THE

AMERICAN MATHEMATICAL SOCIETY

Volume 349, Number 11, November 1997, Pages 4429-4463

S 0002-9947(97)01627-9

\title{
ON COMPOSITE TWISTED UNKNOTS
}

\author{
CHAIM GOODMAN-STRAUSS
}

\begin{abstract}
Following Mathieu, Motegi and others, we consider the class of possible composite twisted unknots as well as pairs of composite knots related by twisting. At most one composite knot can arise from a particular $V$-twisting of an unknot; moreover a twisting of the unknot cannot be composite if we have applied more than a single full twist. A pair of composite knots can be related through at most one full twist for a particular $V$-twisting, or one summand was unaffected by the twist, or the knots were the right and left handed granny knots. Finally a conjectured characterization of all composite twisted unknots that do arise is given.
\end{abstract}

Following Mathieu [Ma], Motegi [Mo] and others, we consider the class of possible composite twisted unknots. In essence, a twisting of a given knot $K$ is constructed by pulling several strands away from the knot, momentarily cutting them, giving them a few full twists, and rejoining the strands to each other as they were before, as illustrated in Figure 1. A knot is composite if it can be described as two simpler knots spliced together. Motegi conjectured, and we show in Section 3, that a twisting of the unknot cannot be composite if we have given the unknot more than a single twist. In Section 2, we also describe all known examples of composite knots that do arise from a single twist of the unknot, and conjecture the list is complete.

But once we have chosen our strands to twist, can we obtain two composite twisted unknots, the first from twisting once one way, the other from twisting once in the other direction? In Section 4, we show that a pair of composite knots can be related through at most a single full twist, or the twist affected only one summand, or that the knots were the right and left handed granny knots, which cannot be twisted unknots by [MY]. The pair of composite knots arising from the same twisting of an unknot would be twistings of one another, related through two full twists, an impossibility, since the only such knots cannot be twisted unknots. Finally we show every composite knot is related to an infinite number of other composite knots through some full twist.

Let us formalize our terms. Let $K$ be a knot in the interior of a standardly embedded solid torus $V$ in $S^{3}$ (Figure 1). Let $\lambda, \mu \subset \partial V$ be the standard longitude, meridian of $V$ in $S^{3}$. For a simple closed curve $\nu \subset \partial V$ such that $[\nu]=[\lambda]+\Delta[\mu]$ in $\mathrm{H}_{1}(\partial V ; Z$ ), let $V(\Delta)=V(\nu)=V \cup$ (solid torus such that meridian lies on $\nu) \cong S^{3}$. We assume that the minimal geometric intersection of $K$ with a meridinal disk of $V$ is at least two. $K_{\Delta}=K \subset V(\Delta)$ then depends on $K$ in $V$ and $\Delta \in Z$. Generally we assume $\Delta>0$, by our choice of orientation of $\mu$. If $K$ is unknotted in $V(0) \cong S^{3}$, we call $K_{\Delta}$ a twisted unknot. If $K$ is a composite knot in $V(0)$ we call $K_{\Delta}$ a twisted composite knot. In general we consider $K_{\Delta}$ to be a $(\Delta, V)$-twisting

Received by the editors August 8, 1994 and, in revised form, September 28, 1995.

1991 Mathematics Subject Classification. Primary 57M25. 

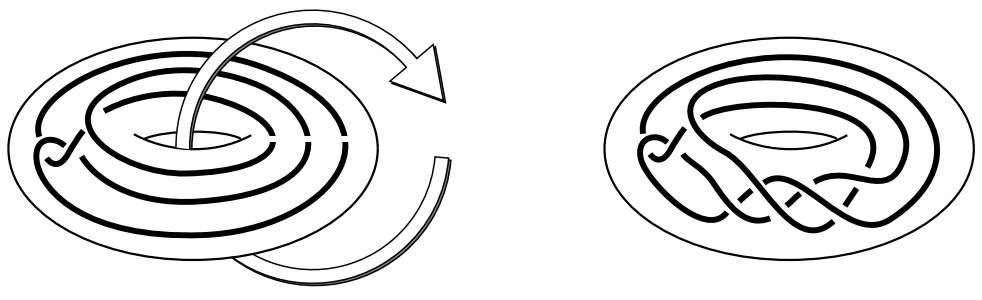

Figure 1. A twisting of a knot

of $K$. We occasionally refer to a $V$-twisting, a $(\Delta, V)$-twisting for any $\Delta$. Mathieu [Ma] described this twisting operation.

A knot $K$ is composite if a splitting sphere can separate $S^{3}$ into balls each containing a single, knotted arc of $K$, a knot summand. In our analysis of composite twisted unknots that arise when $\Delta=1$, there are two cases: either there is a sphere splitting the knot summands that is punctured twice by $\partial V$ or every splitting sphere must be punctured at least four times. With Theorem 2.2 we classify all composite twisted unknots arising from the former. We conjecture that the latter cannot occur.

Motegi [Mo] recently conjectured that each presentation of $K$ in $V$ gives rise to at most one composite twisted unknot and that this $K_{\Delta}$ arises from a single twist of $K$. In [Mo] Motegi proves any such $K_{\Delta}$ has $\Delta \leq 5$; in [T2], Teragaito recently proved that any such $K_{\Delta}$ has $\Delta \leq 2$. In Theorem 3.1 we note that indeed $\Delta=1$ for such $K_{\Delta}$.

In Theorem 4.1 we prove that, but for the single exception of the right and left handed granny knots, $(3,2) \#(3,2)$ and $-(3,2) \#-(3,2)$, given in [HMo], two composite knots can differ by at most one non-trivial twist. (Clearly a twisting that affects only one summand gives rise to another composite knot.) With the results of section 3, this proves Motegi's conjecture.

Very recently, Hayashi and Motegi [HMo] independently proved a theorem similar to Theorem 3.1, with somewhat different techniques than those used here, and proved a weaker version of Theorem 4.1 , namely that a $(\Delta \geq 3, V)$-twisting of a composite knot cannot be composite. Miyazaki and Yasuhara give a sufficient condition for a knot's not being a twisted unknot in [MY] and produce many examples of composite knots that meet this condition.

The techniques of Section 2 are explicit, and are independent of those of Sections 3 and 4 . Our proofs of Theorems 3.1 and 4.1 will rely heavily on the techniques developed in [GL1] and expanded upon in [GL2]. We will assume familiarity with those papers. Loosely speaking, these techniques apply to punctured low genus surfaces, properly embedded in a manifold with torus boundary. If the conditions are right, the techniques produce a negative result, that the surfaces could not have been embedded as desired. Although the lemmas and theorems in [GL1], [GL2], [CGLS] etc. arise from the graphs of intersection of two punctured spheres, we can carefully adapt them to the graphs of intersection of other punctured surfaces. For the proof of Theorem 4.1 we must also introduce loop types to produce ring graphs which give rise to the exceptional case of the granny knots related by 2-twisting.

The author gratefully acknowledges John Luecke's patient and generous guidance. 


\section{The Geometric Setting}

We will first examine a punctured disk arising from the disk bounding an unknot $K$ in $V(0)$, and a punctured sphere derived from a splitting two-sphere for composite $K$ in $V(\Delta)$. The techniques of [GL1] and [GL2] describe the graphs of intersection of such surfaces, the complex formed by their union, and the nature of any manifold that might contain such a complex. As we will see in Section 3 , if $\Delta \geq 2$, such a complex cannot be correctly placed in $S^{3}$, for a contradiction.

Take $V$ in $V(0)=S^{3}$, the complement of a small open neighborhood of an unknot $H$. Thus for any integer $\Delta, V(\Delta)=H(1 / \Delta)$, the $1 / \Delta$ Dehn-surgery on $H$.

Let $D^{*}$ be an embedded disk in $V(0)$ with $\partial D^{*}=K$, such that $D^{*}$ intersects $H$ transversally and minimally. Let $D=D^{*}-\operatorname{int}(N(H))=D^{*} \cap V$, a punctured disk.

Assume $K_{\Delta}$ is composite. Then let $A^{*}$ be an embedded two-sphere in $V(\Delta)$ separating $K$ into two knotted arcs, such that $A^{*}$ intersects $H$ transversally and minimally . Let $A=A^{*}-\operatorname{int}(N(H))=A^{*} \cap V$, a punctured sphere. We can choose $A^{*}$ so that $A$ and $D$ meet minimally and transversally in $V$.

There are three kinds of curves of intersection of $A$ and $D$ in $V$ : arcs with both ends on $\partial V$; simple closed curves of intersection; and either two $\operatorname{arcs} \alpha_{1}, \alpha_{2} \subset A \cap D$ with one end on $\partial V$, the other end in $A \cap K$, or one arc $\alpha_{0} \subset A \cap D$ with both ends in $A \cap K$.

Note that a simple closed curve of intersection $s$ cannot bound a disk in either $A$ or $D$. If $s$ bounds a disk in both $A$ and $D$ then $A$ and $D$ do not intersect minimally. If $s$ bounds a disk in $A$ but not $D$, then $D$ does not intersect $H$ minimally. If $s$ bounds a disk in $D$ but not $A, A$ does not intersect $D$ or $H$ minimally.

We will consider the graphs of only the arcs of intersection of $A$ and $D$ and will take note of the simple closed curves of intersection only in our final geometric arguments. Let $G_{A},\left[G_{D}\right]$ be this graph on $A,[D]$. A pair of these graphs is depicted in Figure 2. Their geometric interpretation may be clearer after examining the constructions in Section 2.

We note that on $\partial V$, the components of $\partial A,[\partial D]$ are parallel and thus share the well-defined slope $1 / \Delta,[0]$. Moreover, each component of $\partial A$ meets each component of $\partial D$ in exactly $\Delta$ points. Thus $G_{A}$ and $G_{D}$ can be described by the nomenclature of pp. 386-387 [GL1].

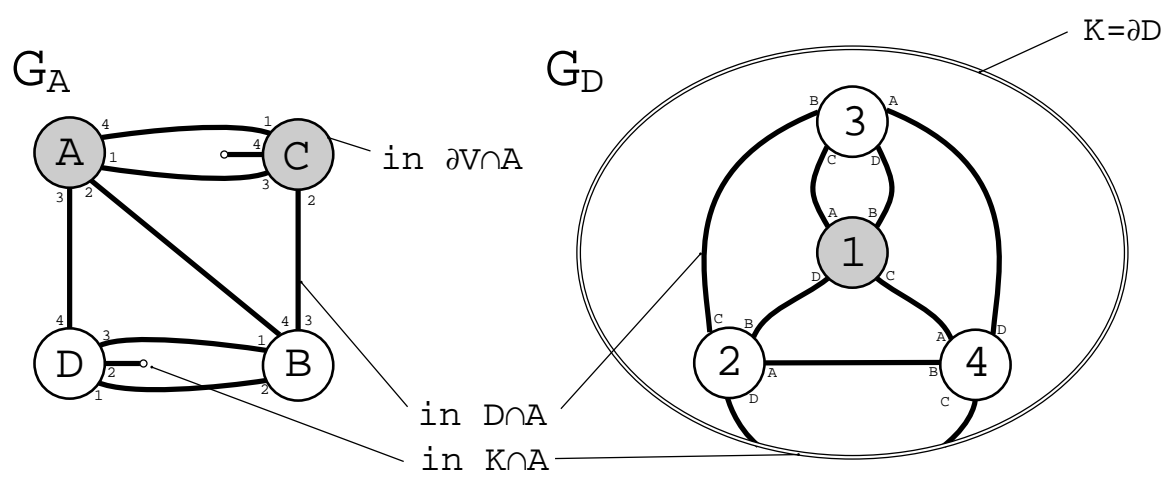

Figure 2. A pair of graphs of intersection 

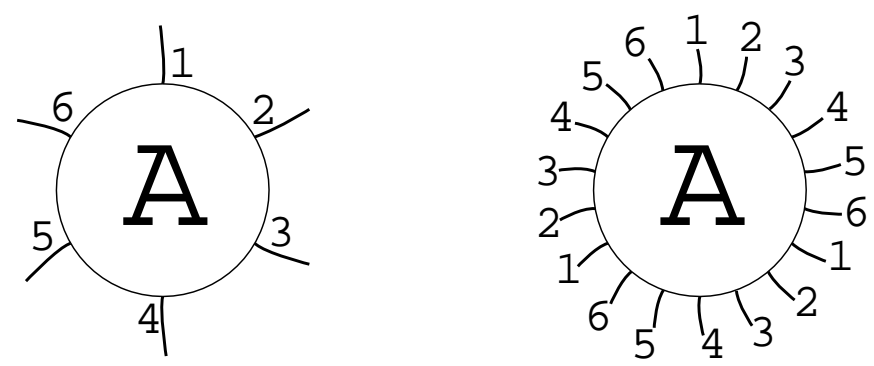

Figure 3. A fat vertex with $\Delta=1$ and one with $\Delta=3$

In particular, $G_{A}$, say, consists of "fat vertices" - the components of $A \cap \partial V$ that are numbered and carry an orientation shown as the shading of the vertices; of edges running between fat vertices- the components of $A \cap D$; and labels at each fat vertex corresponding to the fat vertices of $D$. For our graphs there are also the exceptional edges incident to $K \cap A$, to wit, either $\alpha_{1}$ and $\alpha_{2}$, or $\alpha_{0}$. We stress that $K \subset \partial D$ is not referred to as a fat vertex. Note that since $D$ and $A$ are orientable surfaces properly embedded in an orientable manifold, $G_{D}$ and $G_{A}$ satisfy the parity rule, namely that edges connecting vertices of opposite orientation are incident to labels of the same orientation, and vice versa. As we will point out in Lemma 3.2 below, we can assume there are no boundary parallel arcs in either graph.

In Sections 3 and 4 we will be needing $\Delta>1$ and note that components of $\partial D$ and $\partial A$ will then meet more than once. Accordingly we adopt the conventions of [GL2] with regard to the multiplicity of labels. In particular, a particular label occurs with multiplicity $\Delta$ about a fat vertex. Given a set of labels with order $c$, there are $\Delta c$ representatives of this set at each vertex (Figure 3).

In general we will refer loosely to graphs of this sort, with the accompanying nomenclature, as labeled graphs of intersection.

Faces of $G_{A},\left[G_{D}\right]$ that contain no simple closed curves provide relations in the first homology of $V(0),[V(\Delta)]$ given by generators in $\partial A$ and $D,[\partial D$ and $A]$, as illustrated in Figure 4. Recall that no disk face of either graph contains a simple closed curve of intersection of $A$ and $D$. For $\Delta \geq 2$, combinatorial analysis based on the techniques of [GL2] provides disk faces that imply torsion in the appropriate first homology. [L] gives an expository discussion of these graphs and the techniques used in [GL1], [GL2].

\section{Composite Twisted Unknots}

We will see in Section 3 that any twisted unknot $K_{\Delta}$ with $\Delta>1$ cannot be composite. Here we give a categorization of composite twisted unknots that do in fact arise and conjecture this is complete. Generally, a composite twisted unknot $K_{\Delta}, \Delta=1$, can be characterized by the graphs of intersection $G_{A}$ and $G_{D}$ as constructed above. Recall that $A$ is the portion in $V$ of a separating sphere for $K_{1}$ in $V(1)$, and $D \subset V$ arises from the disk bounded by the unknot $K$ in $V(0)$.

All published examples of composite twisted unknots have a separating sphere punctured twice by $\partial V$, that is, $A$ divides $V$ into two solid tori, each containing 

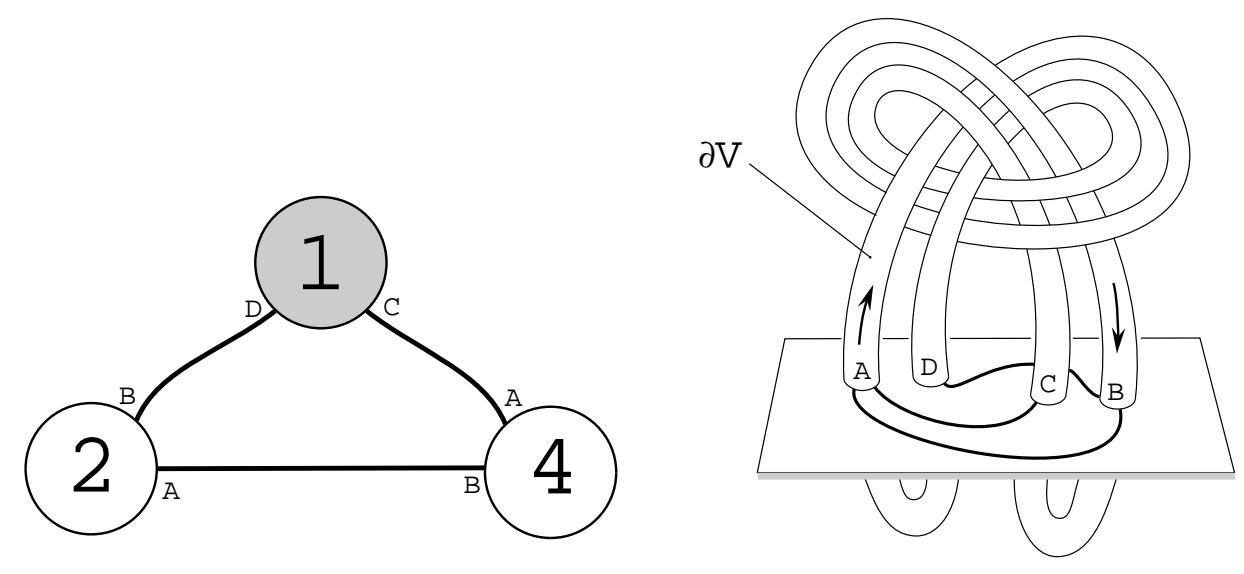

$(A B)(A B)(D C)=1$

FiguRE 4. How the graphs provide relations in the homology

a component of the composite knot. We completely categorize composite twisted unknots with such a separating sphere.

Interestingly, there are pairs of graphs of intersection that might serve as potential $G_{A}$ and $G_{D}$, such that $G_{A}$ has more than four boundary components on $\partial V$, and such that neither graph has obstructions that can be detected by the combinatorics of [GL1] and [GL2]. However, for the time being, all known examples of these graphs have more subtle obstructions, either to properly embedding the induced complex $A \cup D \partial V$ in $S^{3}$, or to producing two non-trivial summands for the twisted unknot $K=\partial D \subset V(1)$. Examples of such graphs are given in Appendix A.

2.1. A Class of Composite Twisted Unknots. An arc $\gamma$ with endpoints on a surface $F$ in a manifold $M$ is called unknotted (with respect to $F$ ) if there is an ambient isotopy of $M$ carrying $\gamma$ to $F$ leaving $\gamma \cap F$ fixed.

A torus one-bridge knot can be described as an arc on the boundary of a standardly embedded solid torus, union an unknotted arc in the solid torus.

Let $k$ be a torus one-bridge knot with presentation on and in a standardly embedded solid torus $V^{\prime}$ in $S^{3}$ as illustrated in Figure 5. In the first case take $\gamma$ to be any arc of $k$ on $\partial V^{\prime}$, in the second let $\gamma$ be the single unknotted arc of $k$ in the interior of $V^{\prime}$. Let $-k_{1}$ be the reflection of a $\left(1, V^{\prime}\right)$-twisting of $k$ (Figure 6); we will see that $K=k \#\left(-k_{1}\right)$ is a composite twisted unknot.

Note that the presentation of $k$ can be isotoped so that $\gamma$ lies on a meridional disk of $V^{\prime}$. Thus we can find an annulus $A \subset \partial V^{\prime}$ parallel to a meridian of $V^{\prime}$ such that $\gamma$ can be isotoped to $A$ in $V^{\prime}$, holding $k \cap \partial V^{\prime}$ fixed, and such that for $A_{0}=\partial V^{\prime}-A$, the components of $k \cap A_{0}$ are simply arcs connecting opposite boundary components of $A$ where each arc misses some meridian of $S^{3}-V^{\prime}$. Note then that $A$ is common to our presentations of both $k$ and $k_{1}$ (and indeed, $k_{\Delta}$ for all $V^{\prime}$-twistings).

We demonstrate that $K=k \#\left(-k_{1}\right)$ is a twisted unknot, by first joining the presentations of $k$ and $-k_{1}$ along $A$ as shown in Figure 7. Embed $A$ on a twosphere $A^{*}$ in $S^{3}$ and take $V$ to be a solid torus standardly embedded in $S^{3}$ such that $V \cap A^{*}=A$. In the illustrations of $A \cup \partial V, V$ has been drawn on the outside 


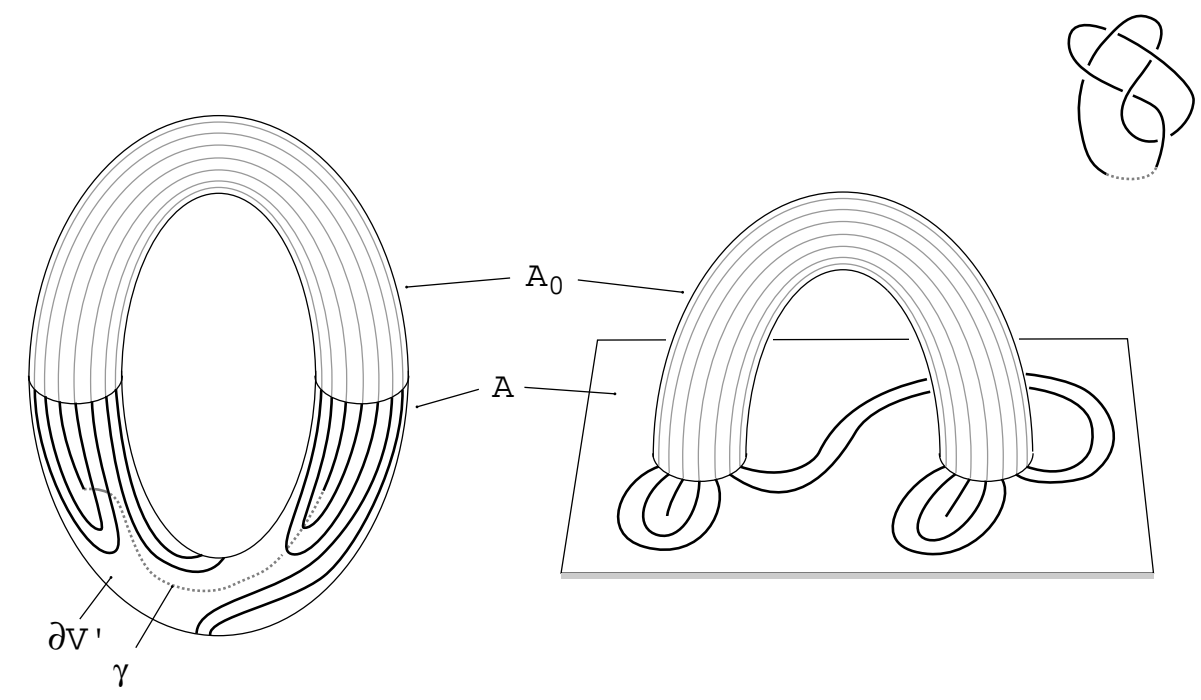

Figure 5. A presentation of a torus one-bridge knot $k$

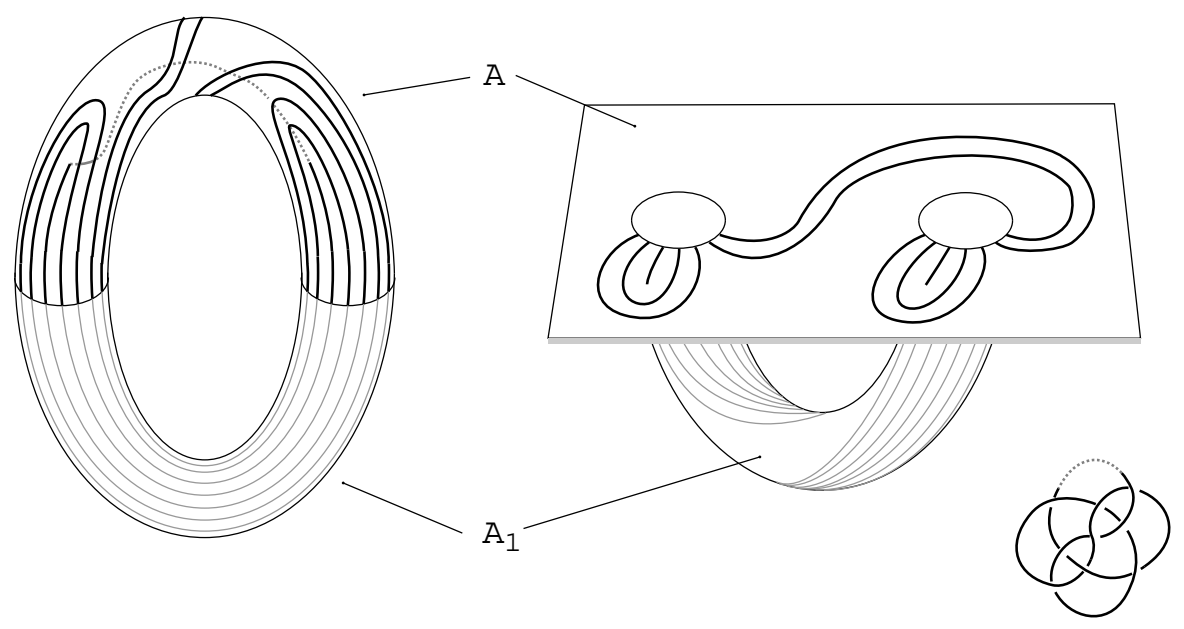

FigURE 6. A presentation of $-k_{1}$

$\partial V$. We consider the boundary of $V$ to be the union of the embeddings of $A_{0}$ and $A_{1}$, separated by $\partial A$. Thus there are two embeddings of $\partial V^{\prime}$ in $A \cup \partial V$, with associated presentations for $k$ and $-k_{1}$, to wit, $A \cup A_{0}$ and $A \cup A_{1}$.

Then by a slight isotopy of the presentations of $k,-k_{1}$ up off $A \cup A_{0}, A \cup A_{1}$, resp., we see that in fact $A^{*}$ separates $K=k \#\left(-k_{1}\right)$, as in Figure 8 . This isotopy of the presentations yields an embedded, punctured disk $D$ with one boundary component $K$ and the other boundary components $(1,1)$-curves on the boundary of $V$. If we cap off $V$ with these curves we produce an embedded disk $D$ in $V(0)$, bounded by $K$ in $V$. And so $K=k \#\left(-k_{1}\right)$ is a twisted unknot in $V(1)$ for all torus one-bridge knots $k$. 

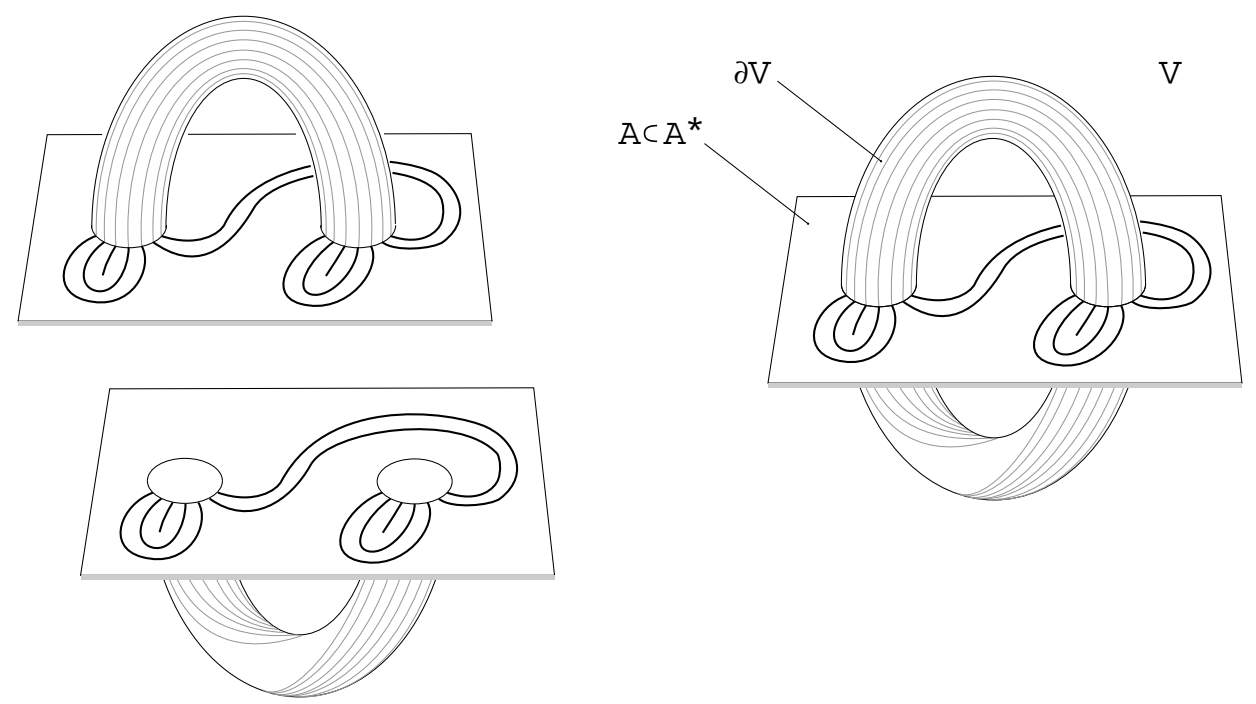

FiguRE 7 . Joining the presentations of $k$ and $-k_{1}$ to obtain $A \cup \partial V$
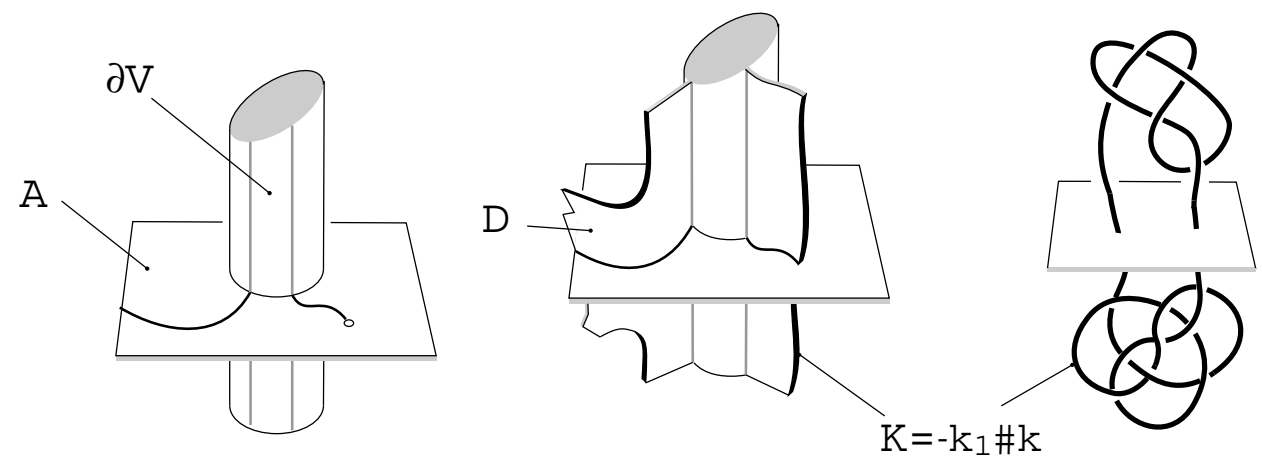

FiguRE 8. Isotoping the presentations of $k$ and $-k_{1}$ to obtain $K$

Note that $G_{A}$ is simply the pattern of $\operatorname{arcs}$ of $k$ on $A$, and that we can easily read off the edges of $G_{D}$ in order (Figure 9). The two faces of $G_{D}$ each present an isotopy from one of the summands of our twisted unknot to $(A \cup \partial V) \cap D$. We have sketched a proof of:

Lemma 2.1. If $k$ is a torus one-bridge knot with presentation in and on a solid torus $V$, then $K=k \#-k_{1}$ is a composite twisted unknot, where $-k_{1}$ is the reflection of a $(1, V)$-twisting of $k$. Moreover, $G_{A}$ has two vertices and $G_{D}$ has one component as constructed above.

Note that this construction accounts for all pairs of connected $G_{D}$, two-verticed $G_{A}$. As shown in Appendix B, most published examples of composite twisted unknots can be presented in this fashion, with the sole exception of the examples given in [T1]. These fall into a slightly broader category, absorbed into Theorem 2.2 . 

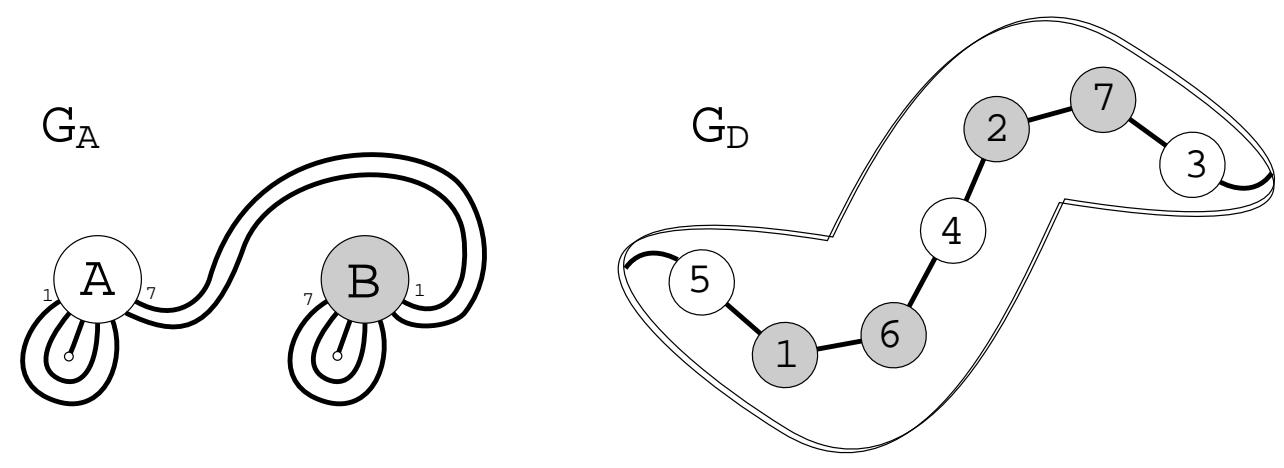

Figure 9. The graphs of intersection for our $K=k \#\left(-k_{1}\right)$

2.2. All Composite Twisted Unknots with $|\partial A|=2$. We define a restricted band sum of a collection of torus 1-bridge knots with disjoint presentations on and in a standardly embedded solid torus $V^{\prime}$ to be a band sum of the knots such that the bands do not intersect each other or the interior of $V^{\prime}$.

Theorem 2.2. If $K$ is a composite twisted unknot with $|\partial A|=2, \Delta=1$, then $K$ in $V(1)$ is of the form $K=S(k) \#-k_{1}$, where

(i) $k$ is a torus 1-bridge knot with presentation in and on a solid torus $V^{\prime}$;

(ii) $-k_{1}$ is the reflection of a $\left(1, V^{\prime}\right)$-twisting of $k$;

(iii) and $S(k)$ is any restricted band sum with respect to $V^{\prime}$ of $k$ with a collection of disjoint $(1,1)$ and $(0,0)$ curves on $\partial V^{\prime}$.

Furthermore, all knots $K=S(k) \#-k_{1}$, as described, are indeed composite twisted unknots.

Note that we can assume that curves on $D$ that are inessential in $\partial V^{\prime}$ are essential in $\partial V^{\prime}-k$. Any innermost inessential curve in $\partial V^{\prime}-k$ with boundary on $D \cap \partial V^{\prime}$ bounds a disk in $V^{\prime}$ that can be used to reduce $D$; similarly, any band sum to an innermost inessential curve in $\partial V^{\prime}-k$ can be retracted.

The following example, from Teragaito [T1], motivates this theorem. This composite twisted unknot $K$ is the sum of a $(p, p+1)$ torus knot and a particular satellite of an arbitrary knot (see Figure 33 in Appendix B). This second summand can also be seen as the restricted band sum of a $(1, p)$ torus knot and a $(1,1)$ curve.

We can parametrize the graphs by $p$. There is a region of $p-1$ pairs of alternating thick and thin arcs and one of $p-2$ thin arcs, and there are other arcs as shown in Figure 10. The thick arcs are a presentation for the torus knots. Further examples are given in Appendix B.

Proof of Theorem 2.2. Each summand of $K_{1}=K \subset V \subset V(1)$ is a band sum of knots that are isotopic to unions of arcs of $A \cap D$ on $A$ and $D \cap \partial V-A$ on $\partial V$ in $V(1)$. This isotopy is simply a retraction of the outermost faces of $G_{D}$ to the outermost arcs of $G_{D}$, with bands between outermost components of $G_{D}$, just as illustrated in Figure 11.

Let $V^{+}, V^{-}$be the closures of the components of $V-A$, and let $V_{1}^{+}, V_{1}^{-}$be the closures of $S^{3}-V^{+}, S^{3}-V^{-}$(Figure 12). Note that $V^{+}, V^{-}, V_{1}^{+}$, and $V_{1}^{-}$are each solid tori. 

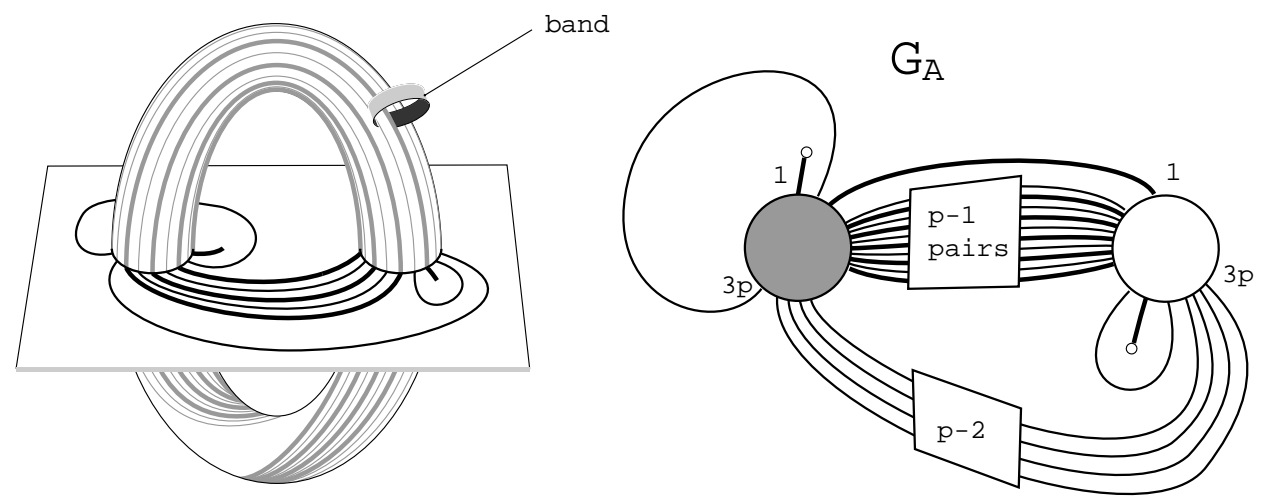

Figure 10. $A \cup \partial V$ and $G_{A}$ for one of Teragaito's examples

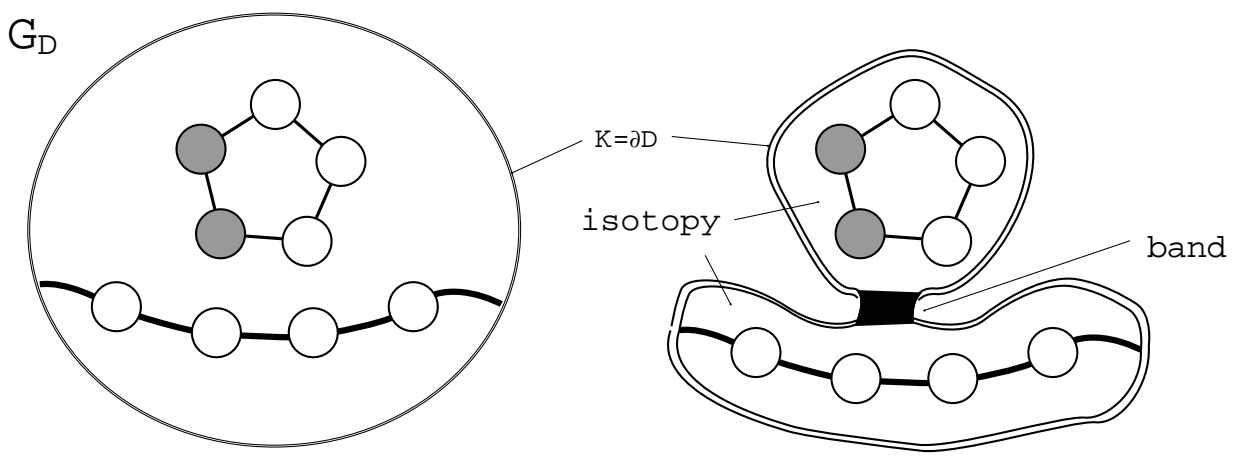

Figure 11. How a band sum arises from a multi-component $G_{D}$
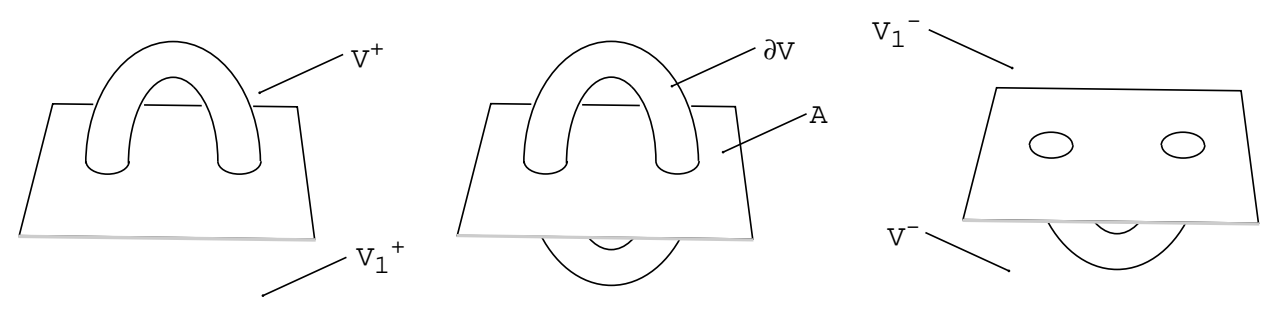

Figure $12 . V^{+}, V^{-}, V_{1}^{+}$, and $V_{1}^{-}$

Let $C^{+}$be the collection of components of $\partial V^{+} \cap D$ on $\partial V^{+}$. Since $\partial D=K$ meets $A$ exactly twice and never meets $\partial V$, these components will include exactly one arc $k^{+}$that may or may not meet $\partial V$ and a possibly vacuous collection of $(0,0)$ and parallel $(p, q)$ curves on $\partial V^{+}$. Similarly, let $C^{-}$be the collection of components of $\partial V^{-} \cap D$ on $\partial V^{-}$.

On $D$ we see that the elements of $C^{+} \cup C^{-}$are isotopic to the boundary components of a neighborhood of $G_{D}$ as illustrated in Figure 13. Since $|\partial A|=2$, each $C_{i}^{+} \in C^{+}$is in one-to-one, onto correspondence with a $C_{i}^{-} \in C^{-}$such that each 
$C_{i}^{+} \cup C_{i}^{-}$is a component of $G_{D}$ on $D$. (For the rest of this proof, we include simple closed curves of intersection in $A \cap D$ in $G_{D}$, with no loss of generality. Thus the $C_{i}^{+} \cup C_{i}^{-}$may be simple closed curves that do not meet $\partial V$ ).

For each $i$, note $C_{i}^{+} \cap C_{i}^{-}$is a collection of arcs on $A$, and, since $\Delta=1,\left(C_{i}^{+} \cup\right.$ $\left.C_{i}^{-}\right)-\operatorname{int}\left(C_{i}^{+} \cap C_{i}^{-}\right)$is a collection of $(1,1)$ curves on $\partial V$, each of which meets $A$ exactly twice.

Thus, in $V(1)$ each $C_{i}^{-}$is the reflection of the $\left(1, V_{1}^{+}\right)$-twisting of $C_{i}^{+}$; that is, $C_{i}^{ \pm}$may be regarded as $-\left(C_{i}^{\mp}\right)_{1}$.

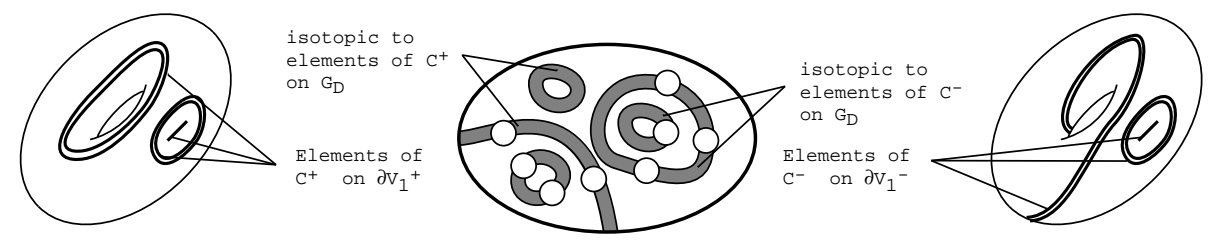

FiguRE 13. Elements of $C^{+}$, and $C^{-}$

So the elements of $C^{-}$are an $\operatorname{arc} k^{-}$, the reflection of a $\left(1, V_{1}^{+}\right)$-twisting of $k^{+}$, and a possibly vacuous collection of $(0,0)$ and parallel $-(p, p \pm q)$ curves on $V^{-}$.

Now if $G_{D}$ has only one component, $C^{+}=\left\{k^{+}\right\}$and $C^{-}=\left\{k^{-}\right\}$. The summands of $K \subset V(1)$ are isotopic to $k^{+}$and $k^{-}$on opposite sides of $A$ and so $K \cong k^{+} \cup k^{-} \cong k \#-k_{1}$ where $k\left[-k_{1}\right]$ is the torus one-bridge knot formed, with presentation in and on $V_{1}^{+}\left[V_{1}^{-}\right]$from $k^{+}\left[k^{-}\right]$by adding a little unknotted arc (the bridge, which is removed when composing the summands) outside of $V^{+}\left[V^{-}\right]$. Note that $-k_{1}$ is the reflection of a $\left(1, V_{1}^{+}\right)$twisting of $k$.

Suppose $G_{D}$ has more than one component; thus there are curves in $C^{+} \cup C^{-}-$ $\left\{k^{+}, k_{1}\right\}$ that are essential on $\partial V_{1}^{+}-k^{+}$or $\partial V_{1}^{-}-k^{-}$. Let $\gamma$ be innermost on $G_{D}$ among all such curves. Suppose then, that $\gamma$ is in, say, $C^{+}$, and is a $(p, q) \neq(0,0)$ curve on $\partial V_{1}^{+}$. Let $F$ be the face of $G_{D}$ incident to $\gamma$, and note that any other boundary components of $F$ are $(0,0)$ curves in $V_{1}^{+}$. By capping these off, with a slight isotopy up off of $\partial V_{1}^{+}$we find a disk in $V^{+}$bounded by $\gamma($ a $(q, p)$ curve on $\left.\partial V_{+}\right)$. But then $(q, p)$ must be $(0,1)$ or $(0,0)$.

So $C^{+}$consists of $k^{+}$, and $(0,0)$ and $(1,0)$ curves on $\partial V_{1}^{+}$; the elements of $C^{-}$, in exact correspondence to those of $C^{+}$, are then $k^{-}$, and $(0,0)$ and $(1,1)$ curves on $\partial V_{1}^{-}$(Figure 13).

Therefore the summands of $K$ can be described: one is the band sum with respect to $V_{1}^{+}$of a torus one bridge knot $k$ with presentation on and in $V_{1}^{+}$, with $k \cap \partial V_{1}^{+} \cong k^{+}$, and a possibly vacuous collection of $(0,0)$ and $(1,0)$ curves on $\partial V_{1}^{+}$; the other summand is the band sum with respect to $V_{1}^{-}$of $-k_{1}$, the reflection of a $V_{1}^{+}$twisting of $k$ with $-k_{1} \cap \partial V_{1}^{-} \cong k^{-}$, and a possibly vacuous collection of $(0,0)$ and $(1,1)$ curves on $\partial V_{1}^{-}$.

We next show that any composite twisted unknot of this form can actually be reduced to the form $S(k) \#-k_{1}$ as described in the hypothesis of the theorem.

If $G_{D}$ has more than one component, $G_{D}$ has disk faces. By arguments above, the boundary of such a disk face must be either a $(1,0)$ curve on $\partial V_{1}^{+}$or a $(0,0)$ curve on $\partial V^{ \pm}$essential in $\partial V^{ \pm}-k^{ \pm}$.

1. Suppose there is a disk face $F$ of $G_{D}$ bounding a $(1,0)$ curve in $\partial V_{1}^{+} . F$ is a meridional disk of $V^{+}$and all band sums in $V^{+}$are actually contained in the 
ball $B=V^{+}-\operatorname{int} N(F)$. All the curves of $C^{+}$to which $k^{+}$is bandsummed are thus inessential in $\partial B-k^{+}$, and can be retracted, since any innermost such curve bounds a disk in $\partial B$ missing $D$. Thus the summand of $K$ in $V^{+}$ could only be a torus one bridge knot, by abuse of notation denoted $-k_{1}$. The other summand can be described as the special band sum $S(k)$, of the form given in the statement of the theorem.

2. Suppose that there is a disk face of $G_{D}$ bounding a $(0,0)$ curve in $\partial V_{1}^{ \pm}$, essential in $\partial V_{1}^{ \pm}-k^{ \pm}$. Then the summand of $K$ in $V^{ \pm}$is contained in a ball, bounded by our disk face of $G_{D}$ and the disk bounded by the $(0,0)$ curve in $\partial V_{1}^{ \pm}$. Again, any band sum, with respect to a ball, of an arc to a number of simply closed curves on the boundary of this ball, must be trivial. So in this case, the summand of $K$ in $V^{ \pm}$is a torus one bridge knot $-k_{1}$, and the other summand is a band sum with respect to $V_{1}^{\mp}$ of $k$ and a collection of $(1,(1 \mp 1) / 2)$ curves in $\partial V_{1}^{\mp}$ and $(0,0)$ curves essential in $\partial V_{1}^{\mp}-k^{\mp}$.

If this band sum is in $V^{-}$, the sum is now in the form given in the statement of the theorem.

3. Assume then that the sum is in $V^{+}$. That is, one summand of $K$ is the restricted band sum with respect to $V_{1}^{+}$of a torus one-bridge knot $k$ with presentation in and on $V_{1}^{+}$and a collection of $(1,0)$ and $(0,0)$ curves on $\partial V_{1}^{+}$, such that all $(0,0)$ curves are essential in $\partial V_{1}^{+}-k$. We will next isotope the $(1,0)$ curves in $V^{+}-K$ so that the summand $K$ is presented as the band sum of $k$ with $(0,0)$ curves, whence $K$ will be in the form $S(k) \#-k_{1}$.

4. If $k$ is band summed to $(1,0)$ curves, there is a face $C$ of $G_{D}$ with one boundary component a $(1,0)$ curve on $\partial V_{1}^{+}$, the others all $(0,0)$ curves essential in $\partial V_{1}^{+}-k$. If this second class of boundary curve is vacant, then this face is a meridional disk of $V^{+}$and the band sum is trivial as above. Otherwise, we can use this face of $G_{D}$ to present any $(1,0)$ curve as the band sum of $(0,0)$ curves. We simply slide any $(1,0)$ used in the band sum, beginning with the closest, along the boundary of a neighborhood of $C$ as illustrated in Figure 14. And so any composite twisted unknot with $\Delta=1$ and $|\partial A|=2$ is of the form $S(k) \#-k_{1}$ as stated in the theorem.
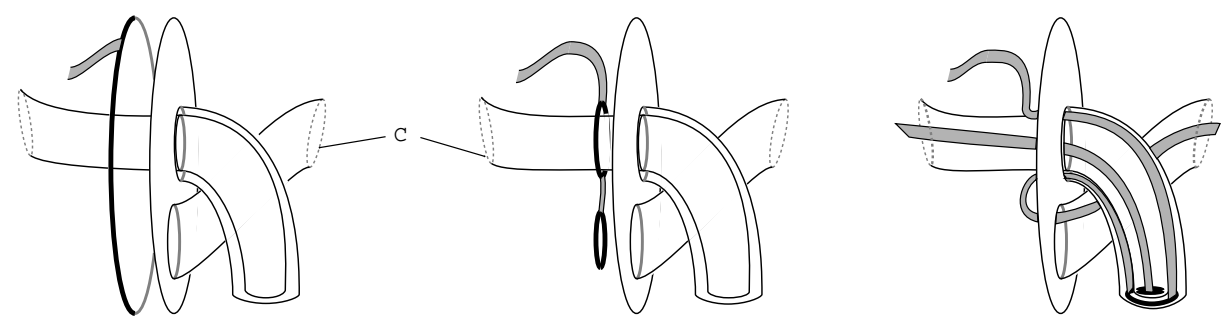

FiguRe 14. Sliding curves

We now turn to the converse and for any knot of the form $S(k) \#-k_{1}$ produce a corresponding complex $A \cup D \cup V$. Let $k$ be a torus one bridge knot presented in and on a standardly embedded solid torus $V^{\prime}$, and let $G_{D}^{\prime}$ be the graph presenting $k \#-k_{1}$ as a composite twisted unknot, as constructed in Section 2.1. In this construction, let $V^{\prime}=V_{1}^{-}$, and let $-k_{1}$ be presented in and on the solid torus $V_{1}^{+}$. There are $(1,1)$ and $(0,0)$ curves on $\partial V_{1}^{-}$that miss $k \cap \partial V_{1}^{-}$. These curves 
correspond to $(1,0)$ and $(0,0)$ curves on $\partial V_{1}^{+}$that miss $-k_{1} \cap \partial V_{1}^{+}$. Together, these curves have an induced placement on $\partial V \cup A$. We are free to cap off the curves on $\partial V_{1}^{+}$with disks in $V^{+}$; we can form any band sum we please between the curves on $\partial V_{1}^{-}$and $k$ in $V^{-}$.

We construct $D$, a punctured disk in $V$, with one boundary component any knot $S(k) \#-k_{1}$ and the other components $(1,1)$ or $(0,0)$ curves on $\partial V$, by piecing together the caps in $V^{+}$and bands in $V^{-}$, and small interval products above each element of $C^{ \pm}$in $V^{ \pm}$.

The graph $G_{D}$ is thus composed of $G_{D}^{\prime}$ banded with as many copies of $G_{0}$ (for $(0,0)$ summands) and $G_{1}$ (for $(1,1)$ summands) as we wanted curves to which to bandsum (Figure 15). Note that the placement of $k$ on $\partial V^{+}$completely determines the number of vertices in each component of $G_{D}$, the relative parity of the vertices in each component, and the order of the labels of the vertices in each component.
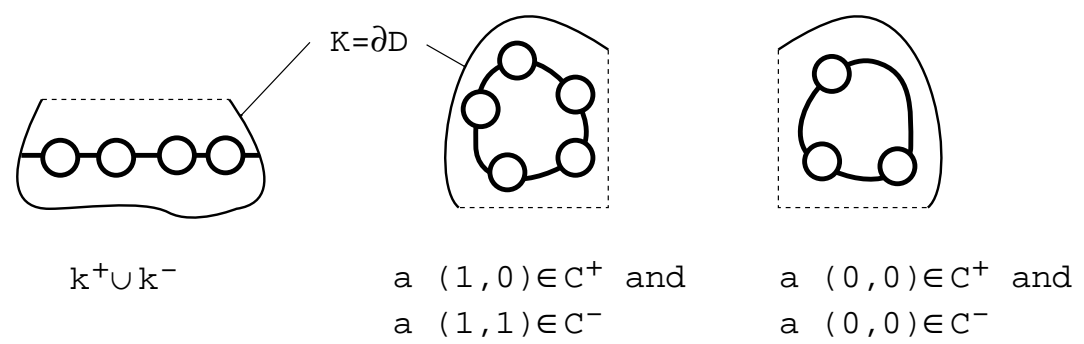

Figure 15. Pieces of $G_{D}$

Thus, with Theorem 3.1 and Corollary 4.2, Theorem 2.2 classifies all composite twisted unknots, up to the conditions of the following conjecture. Teragaito makes this same conjecture in [T2].

Conjecture 2.3. If $K$ is a composite twisted unknot then there is some separating sphere for $K$ in $V(1)$ that meets $\partial V$ exactly twice.

\section{Not Composite Twisted Unknots}

We set out to prove:

Theorem 3.1. If $\Delta \geq 2$, then $K_{\Delta}$ cannot be a composite, twisted unknot.

Recall the setting put forth in Section 1. For the proof of the theorem, we will replace $A$ with a punctured torus so that all of our arcs of intersection have both ends on $\partial V$. Let $T^{*}$ be the torus formed by tubing $A^{*}$ along $K$ in one direction as pictured in Figure 16. That is, take $N$ to be one of the components of $\partial N(K)-A^{*}$ then $T^{*}=\left(A^{*}-\operatorname{int}(N(K))\right) \cup N . T^{*}$ is sometimes known as a swallow/follow torus since it swallows one summand and follows the other. Note $T^{*} \cap K=\emptyset$. Let $T=T^{*} \cap V=(A-\operatorname{int}(N(K))) \cup N$.

Recall the definition of the $\operatorname{arcs} \alpha_{1},\left[\alpha_{2}\right]$ with one end on $\partial V$, the other end in $A \cap K$ as given in Section 1. The curves of intersection of $T$ and $D$ in $V$ are derived from those of $A$ and $D$ (Figure 17). The arcs not meeting $K$ and the simple closed curves of intersection are unaffected by the transition. However, we obtain either an arc $\alpha$ running between the ends of the $\alpha_{i}$ on $\partial V$, derived from $\alpha_{1}$ and $\alpha_{2}$, or a 

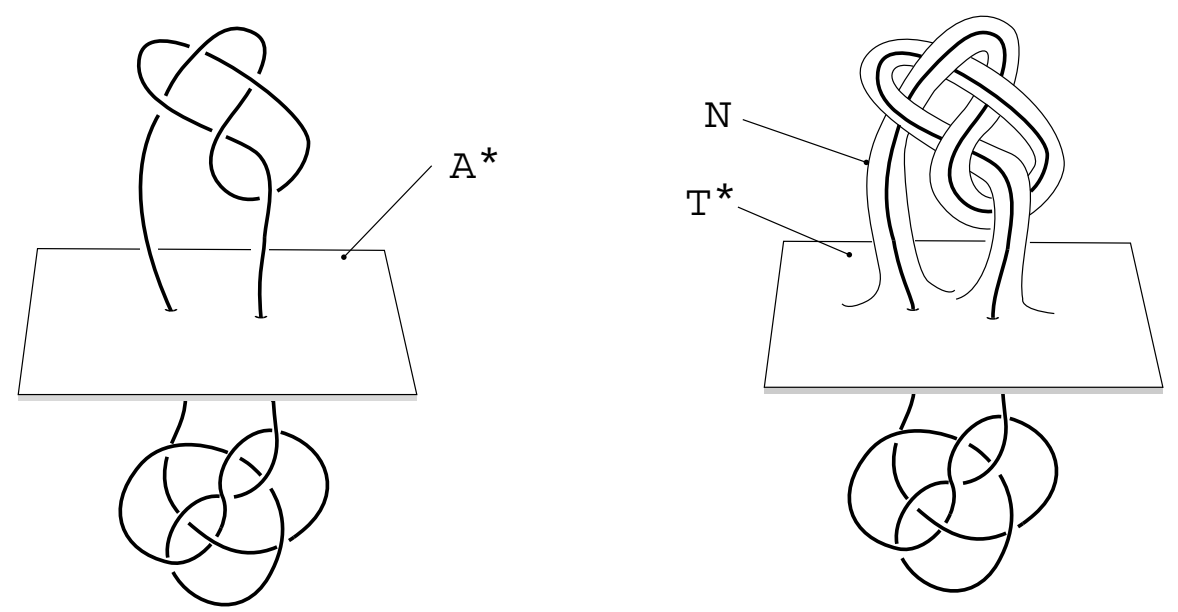

FiguRE 16. Creating a "swallow/follow" torus

simple closed curve $\alpha^{\prime}$, derived from $\alpha_{0}$. Figure 17 illustrates this first case for a graph with $\Delta=1$.

If $\alpha$ arises, let $\alpha^{\prime}=\emptyset$ and note that by our choice of $N$ we determine which of the two outside faces of $G_{D}$ will be closed off by $\alpha$ during this construction.

If $\alpha^{\prime}$ arises, take $\alpha=\emptyset$ and note that if $\alpha^{\prime}$ bounds a disk in $D$, then $A$ could not have separated $K=\partial D$ into non-trivial summands. Furthermore, by construction, $\alpha^{\prime}$ must be non-separating on $T$.

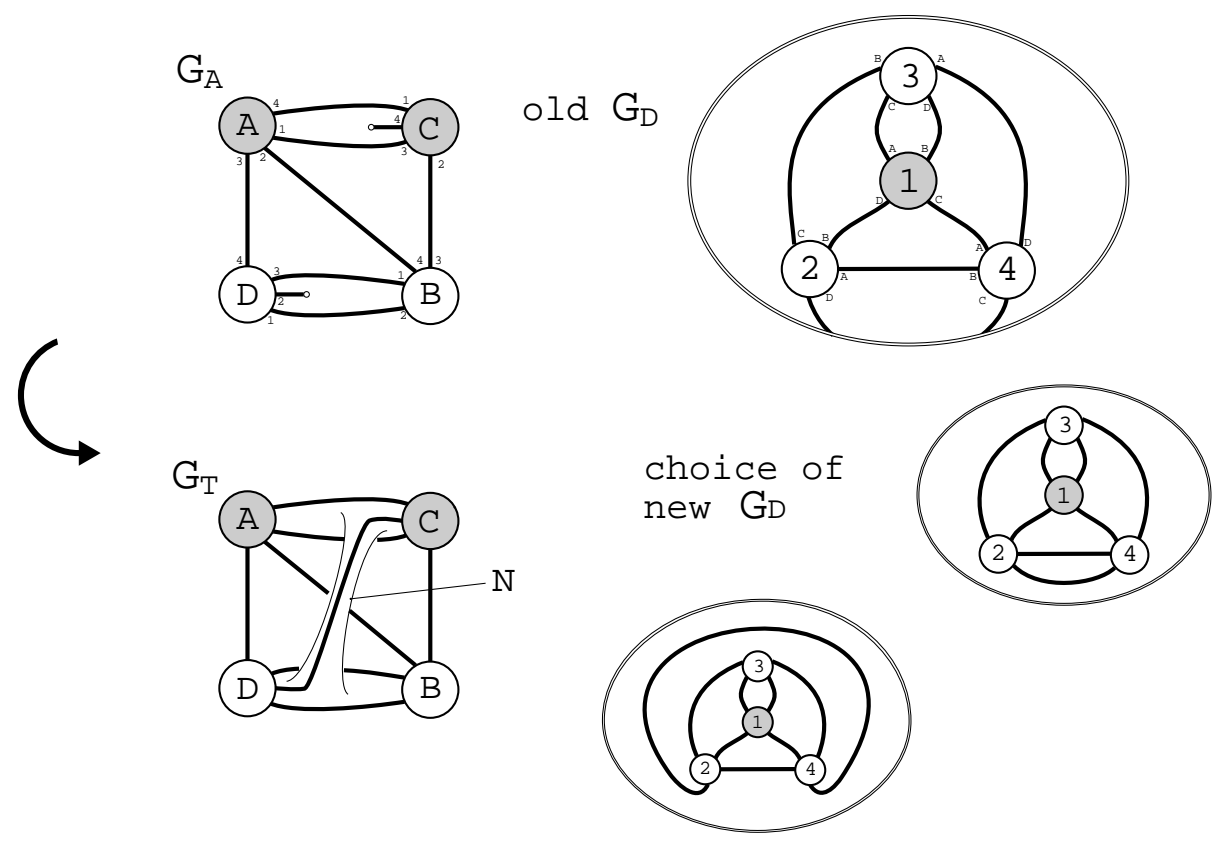

FiguRE 17. Constructing the new graphs of intersection 
Again, we will consider the graphs of only the $\operatorname{arcs}$ of intersection of $T$ and $D$ and will take note of the simple closed curves of intersection only in the final geometric argument. We let $G_{T},\left[G_{D}\right]$ be this graph on $T$, $[D]$, suppressing our previous definition of $G_{D}$. Just as in Section 1, these graphs are labeled graphs of intersection as described in [GL1].

However we note there are some differences in the nature of the surfaces used here and those used in [GL1] and [GL2]: a component of $\partial D$ is not in $\partial V$, and $T$ is not a punctured sphere. However, most of the combinatorial arguments are local in nature and will not be affected, and many others will suffer only a change in Euler characteristic. The core of the combinatorial argument is induction within an inessential curve on one of our surfaces. In order to insure that the curve separates and that the induction is finite, the induction must take place on $D$, working in away from $K$.

Let $\hat{T},[\hat{D}]$ refer to the (abstract) torus, [disk] formed by contracting each fat vertex of $G_{T},\left[G_{D}\right]$ to a point. Finally, let $p, q$ be the number of fat vertices on $G_{T}$, $G_{D}$, respectively. Clearly $q>0$, or $D^{*}$ would lie completely within $V$ and $K$ would be both an unknot and a non-trivial composite knot in $V(\Delta)$, a contradiction to the unique decomposition of knots. Similarly, if $p=0$, then $A^{*}$ would lie within $V$ and $K$ is both a non-trivial knot and an unknot within $V(0)$, a contradiction. Consequently, $p \geq 2$ since $A^{*}$ separates $V(\Delta)$ and $p$ is even.

For a graph $G$ of properly embedded arcs on a punctured surface $F$ we call a component $c$ of $F-G$ a one-sided face if $c$ is a disk bounded by a single arc of $G$ and some arc in $\partial F$.

Lemma 3.2. By suitable choice of $N$ in the construction of $T$, we can assume there are no one-sided faces in $G_{T}$ or $G_{D}$.

Proof. Suppose there were such a face $c$ on either $T$, or $D$. If $\alpha$ is not in $\partial c$ then if $c$ lies on, say, $T, c$ provides an isotopy of $D$ in $V(0)$ reducing the number of intersections of $D$ with $H$, contrary to our construction of $D$.

We are left with the possibility that $c$ lies on $D$ and is partially bounded by $\alpha$. Momentarily consider $S$, the punctured two-sphere, formed by abstractly adding a disk to $D$ along $K$. Then $G_{D}$ lies on $S$ in a natural fashion. Unless $G_{D}$ consists of only $\alpha$ and a single vertex, $\alpha$ can only bound at most one one-sided face on $S$. By choice of $N$ in the construction of $T$, we can arrange that this one-sided face does not lie on $D$.

If $G_{D}$ does in fact consist only of $\alpha$ and a single vertex, then $D$ is an embedded annulus in $V(\Delta)$ with one boundary component $K(\Delta)$ a composite knot and the other a curve on $\partial V$, an unknotted solid torus, giving a contradiction, for torus knots cannot be composite.

We move to the combinatorics developed in [GL1] and [GL2]. As mentioned in Section 1, we seek special disk faces in the graphs of intersection that provide relations in the first homology of the complex formed by our surfaces, the twisting torus and an appropriate choice of meridians in the complement of the twisting torus. Scharlemann cycles are disk faces whose corners all lie on the same label pair, at vertices of the same parity. They thus provide a cyclic relator; this relator is non-trivial since we are assured that all faces have more than one corner, i.e. there are no one-sided faces. A type is defined in [GL1] as a particular kind of relation; a disk face represents a type if it relates the relation. If we find disk face 
representatives for all types, $[\mathrm{P}]$ demonstrates torsion in the appropriate complex. In essence, we seek all types on $G_{T}$ or a Scharlemann cycle on either $G_{T}$ or $G_{D}$. For a lucid expository discussion, see [L].

We take notation as in [GL1] and [GL2]. In particular recall the conventions regarding the multiplicity of labels given before Lemma 2.5 [GL2], and the definition of an $x$-web, given before Lemma 2.3 of [GL2]. See Figures 3 and 18 in Sections 1 and 4 . We do assume that all vertices not belonging to a given great $x$-web lie in a single component of the complement of the web.

Lemma 3.3. Take $G_{T}, G_{D}$ as above. Let $L$ be a subset of the labels on $T$, with $|L| \geq 2$. Let $\tau$ be a non-trivial L-type. Take $\chi_{T}=\chi(\hat{T})$ and let $c=|C(t)|$ for $t$ a star representing $\tau$. Let there be $r$ disk face representatives of $\tau$ on $G_{T}$ and $s$ switch edges. Then $r+s \geq(\Delta c-1) p+\chi_{T}$.

Proof. This is a simple restatement of Lemma 2.3.2 of [GL1]. Note only the following changes: the definition of $i$ yields $\Delta c-1=i$; and the Euler characteristic of the surface is 0 rather than 2. Keeping track of these changes, the proof remains the same.

Lemma 3.4. Take $T, D$ as above, with $\Delta \geq 2$. For a particular L-type $\tau,|L| \geq 2$, take $t$ a star representing $\tau$ and $L(t)=L$. If all elements of $C(t)$, and all elements of $A(t)$ have the same parity then there is either a disk face representative of $\tau$ in $G_{T}(L)$, or there is an $x$-web $\Sigma$ in $G_{D}$ such that the set of vertices of $\Sigma$ is a subset of either $C(t)$ or $A(t)$.

Proof. Suppose there is a type $\tau$, (with star $t,[t]=\tau$ ), not represented in $G_{T}(L)$ and let $s$ be the number of switch edges in $\Gamma_{T}^{*}(t)$. Then by the above lemma, $s \geq(\Delta c-1) p$, i.e. there are at least $(\Delta c-1) p / 2$ edges whose ends are both incident to, say, elements of $C(t)$. Thus at least $(\Delta c-1) p$ of the $\Delta c p$ clockwise switches of $G(L)$ are incident to switch edges, and so there is some vertex $x$ of $G_{T}$ such that at least $(\Delta c-1)$ switch edges are incident to the clockwise switches at $x$.

Then as in Lemma 2.5 in [GL2], we see that on $G_{D}$ there is a fat vertex $y_{0} \in C(t)$ such that for $y \in C(t)-y_{0}$ every $x$-edge in $G_{D}$ incident to $y$ has both ends incident to fat vertices in $C(t)$. All but one $x$-edge at $y_{0}$ connects $y_{0}$ to a fat vertex in $C(t)$. Since all elements of $C(t)$ are parallel, these edges form an $x$-web.

Lemma 3.5. Assume $\Delta \geq 2$. If $G_{D}$ contains a great $x$-web $\Sigma$, let $U$ be the component of $\hat{D}-\Sigma$ such that all the vertices of $C=\hat{D}-U$ have the same parity. Then $G_{D}$ contains two Scharlemann cycles in $C$.

Proof. Each component of $C-\Sigma$ is bounded by a great $x$-cycle and thus contains a Scharlemann cycle by Lemma 2.2 of [GL2]. Since $\Delta \geq 2$ there are at least two such components. Note that the proof rests on $T$ separating $V$.

We will now devote some energy to the following lemma, modeled closely in statement, proof and intent on Lemma 2.8.1 of [GL1] and Proposition 3.1 of [GL2]. With the geometric interpretation of this lemma provided by Lemma 3.9 the proof of Theorem 3.1 will be complete.

Proposition 3.6. Take $T$ and $D$ as above. Let $C$ be a disk in $\hat{D}$ such that $G_{D} \subset$ $\operatorname{int}(C)$ or let $C$ be a disk bounded by an $x$-web $\Sigma$. Let $L$ be the vertices of $G_{D}-\Sigma$ in int $(C)$. Assume $\Delta \geq 2$. Then $G_{D}$ contains two Scharlemann cycles, or $G_{T}$ 
contains one Scharlemann cycle, or each L-type $\tau$ is represented by some face of $G_{T}(L)$.

Note that if we take $C$ such that $G_{D} \subset \operatorname{int}(C)$, we take $\Sigma=\emptyset$.

We will proceed by looking for a disk face representative of each $L$-type $\tau$ on $G(L)=G_{T}(L) \subset G_{T}$. If a given $L$-type $\tau$ is not represented, we find an $x$-web in the interior of $C$. In this way, we eventually find all $L^{\prime}$-types in $G\left(L^{\prime}\right)$ for a particular set of labels $L^{\prime} \subset L$ or we arrive at a great $x$-web on $D$ - that is, an $x$-web bounding a disk in $\hat{D}$ containing no vertices of $G_{D}$ other than those of the $x$-web itself.

Lemma 2.4.1 and its relatives in 2.6 and 2.7 of [GL1] are used to demonstrate that if all $L^{\prime}$-types are represented in $G\left(L^{\prime}\right)$ for $L^{\prime}$ derived as above, then a disk face of $G(L)$ represents $\tau$. Although [GL1] considers graphs on planar surfaces and we are looking for representatives of types on a torus, these lemmas and arguments take place within a particular disk face of the graph and so are local in nature. Thus we will invoke them as needed.

Lemma 3.7. $|L|>1$, or $G_{T}$ contains a Scharlemann cycle, or $G_{D}$ contains two Scharlemann cycles.

Proof. Assume $|L| \leq 1$. Then either $\Sigma$ is a great $x$-web or $L$ contains exactly one vertex $v$, antiparallel to those of $\Sigma$. In the first case there are two Scharlemann cycles in $G_{D}$ by Lemma 3.5.

In the second case, note that any edge of $G_{D}$ with both ends incident to $v$ must bound a one-sided face on $D$, since $L=1$ and $C$ is a disk. Since there are no one-sided faces in $G_{D}$, every edge incident to $v$ must connect $v$ to a vertex of $\Sigma$. By the parity rule, every edge with label $v$ on $G_{T}$ must connect parallel vertices of $G_{T}$. Let $\Lambda$ be the subgraph of $G_{T}$ consisting of all vertices of $G_{T}$ along with all edges of $G_{T}$ with label $v$. Note that the vertices of each component of $\Lambda$ are all parallel; there must be at least two such components since $T$ separates $V$.

There exists a component $c$ of $\Lambda$ within an open annulus $a \subset T$ such that $\Lambda \cap a=c$. This annulus may or may not be essential in $T$. Let $G=$ vertices and edges of $G_{T}$ completely contained within $a$. That is, $c$ is a subgraph of $G$. We will consider the faces of both $c$ and $G$ to be only those faces of $G_{T}$ completely contained within $a$; i.e. all faces of $c$ and $G$ are disks and the interior of no face of $G$ intersects an edge of $G_{T}$.

Claim 1. Any digon in $c$ induces a Scharlemann cycle on $G$.

Proof. Without loss of generality, choose an innermost digon $\delta$ on $c$ with edges $e_{1}$, $e_{2}$, and vertices $x, y$. Let $q$ be the number of vertices of $G_{D}$.

If both edges are not incident to $v$ at the same vertex, $\delta$ is a great $v$-cycle within a disk and so by Lemma 2.6.2 of [CGLS], $G$ contains a Scharlemann cycle.

If both edges are incident to label $v$ on, say, $x$, there exists at least $q-1$ edges of $G$ in the interior of the digon, all of which connect $x$ to $y$. On $y$, then, we see $q-1$ consecutive labels in int $\delta$, one of which must be $v$ since $v$ could not have been the label of either $e_{i}$ at $y$ by the parity rule. But then there is an $v$-edge in $\delta$ and $\delta$ was not innermost in $\Lambda$.

Claim 2. Any trigon in $c$ induces a Scharlemann cycle on $G$.

Proof. Without loss of generality, choose an innermost trigon $t$ with vertices $x_{1}, x_{2}$, $x_{3}$, and edges $e_{12}, e_{23}, e_{31}$, with $e_{i j}$ running between $x_{i}$ and $x_{j}$. If the $e_{i j}$ do not 
form a great $v$-cycle, after relabeling we have that $e_{12}, e_{23}$ are both incident to $v$ at $x_{2}$. Now there are at least $q+1$ edges in $t$ meeting $x_{2}$ (including $e_{12}, e_{23}$ ). Then there are at least $(q+1) / 2$ edges parallel to, say, $e_{12}$. Thus there is some label $w$ such that there are $w$-edges $e_{1}, e_{2}$, parallel to $e_{12}$.

The proof of case 1 can be applied to the digon bounded by $w$-edges $e_{1}, e_{2}$, which thus contains a Scharlemann cycle in $G$.

Claim 3. There exists a trigonal or digonal face of $c$.

Proof. Abstractly consider $c^{*}$, a graph on the 2 -sphere formed by adding two disks to the boundary of $a . c^{*}$ may now have up to two one-sided faces, each with an edge parallel to the boundary of $a$. So we will show that $c^{*}$ has at least three faces that are either one-sided, a digon or a trigon, giving at least one digonal or trigonal face for $c$. Let $f_{k}$ be the number of $k$-gonal faces of $c^{*}, f=\Sigma f_{k}, e$ be the number of edges of $c^{*}, v$ be the number of vertices of $c^{*}$. Assume for contradiction that $f_{1}+f_{2}+f_{3} \leq 2$. Note $e=\Delta v$ by the parity rule, and $2 e=\Sigma k f_{k} \geq 2+4(f-2)$. Since $v+f-e=2$, we have $e / \Delta+(e+3) / 2-e \geq 2$ or $e(1 / \Delta+1 / 2-1) \geq 1 / 2$. However this is a contradiction since we now invoke that $\Delta \geq 2$ and so $(1 / \Delta+1 / 2-1) \leq 0$.

Thus in the second case of the lemma there is a trigonal or digonal face of $c$ and consequently a Scharlemann cycle on $G_{T}$.

Lemma 3.8. Assume the hypotheses of Proposition 3.6. Then there is a face of $G_{T}(L)$ representing the trivial L-type $\tau$ or there are two Scharlemann cycles in $G_{D}$.

Proof. Let $J \subset L$ be the set of vertices in $C$ of opposite sign from those of $\Sigma$, or if $\Sigma=\emptyset$, let $J$ be the set of, say, + vertices in $C$. Assume $G_{D}$ does not contain two Scharlemann cycles, and hence no great $x$-web. Thus $J \neq \emptyset$.

Case 1 . Suppose at each vertex $x$ of $G_{T}$ we can take two edges $e_{1}(x), e_{2}(x)$ incident to labels in $J$ at $x$ and connecting $x$ to a parallel vertex. Note that for any such edge, the other label is not in $J$ by the parity rule so there are $2 p$ such edges if there are $p$ vertices of $T$. Let $F$ be the collection of faces of the subgraph $\Lambda$ of $G_{T}$ formed by these edges and the vertices of $G_{T}$. Then $p-2 p+\Sigma_{f \in F} \chi(f)=\chi(\hat{T})=0$. Recall that $p>0$ and so there must be some $f \in F$ with $\chi(f)>0$. That is, there is a disk face in this subgraph incident only to parallel vertices. Now any face of $G_{T}(L)$ contained in $f$ is also a disk face incident to parallel vertices and thus represents the trivial $L$-type.

Case 2. This follows the proof of Case 1.a of Lemma 3.3 of [GL2]. Suppose there exists a vertex $y$ of $G_{T}$ such that at most one of the edges incident at labels in $J$ to $y$ connects $y$ to a parallel vertex (possibly $y$ itself). Let $v_{0}$ be the label at $y$ of the edge connecting $y$ to a parallel vertex ( $\left\{v_{0}\right\}$ may be empty). Then on $G_{D}$, for all vertices $v \in J-\left\{v_{0}\right\}$, all edges incident to the label $y$ at $v$ connect $v$ to parallel vertices, by the parity rule. Moreover, all but at most one of the edges incident to $y$ at $v_{0}$ connect $v$ to parallel vertices. All these parallel vertices must be elements of $J$ since $J$ is precisely the set of vertices parallel to $v, v_{0}$ within components of $C-\Sigma$. Thus there is a $y$-web $\Sigma_{0}$ between vertices of $J$. Let $C_{0} \subset \hat{D}$ be the disk bounded by $\Sigma_{0}$ in $\operatorname{int}(C)$. Let $L_{0} \subset L$ be the set of vertices of $G_{D}$ in $\operatorname{int}\left(C_{0}\right)$ Then since $\left|L_{0}\right|<|L|$ we may assume by induction that there exists two Scharlemann cycles on $G_{D}$ or the trivial $L_{0}$-type is represented by some disk face of $G_{T}\left(L_{0}\right)$. Any such disk face in $G_{T}\left(L_{0}\right)$ is incident only to parallel vertices of $G_{T}$ and so any face of $G_{T}$ contained within this face will represent the trivial $L$-type. 
Proof of Proposition 3.6. This is simply the proof of Proposition 3.1 in [GL2], using Lemma 3.4 for Theorem 2.5 [GL2], Lemma 3.7 for Lemma 3.2 [GL2] and Lemma 3.8 for Case 1 of the proof of Proposition 3.1 [GL2]. Recall the comments immediately following the statement of Proposition 3.6. Note that the inductive $D$ used in [GL2] is our $C$.

Next we give the geometric interpretation of the results of Proposition 3.6. This will complete the proof of Theorem 3.1.

Lemma 3.9. If $D, T$ are constructed from a composite twisted unknot as above, $G_{T}$ cannot represent all types and $G_{D}$ cannot contain two Scharlemann cycles.

Proof. By the proof of Proposition 3.6, either all types are represented by disk faces of $G_{T}$ or $G_{D}$ contains two Scharlemann cycles. (Recall that Scharlemann cycles represent all types.) In the first case, since there are no inessential simple closed curves of intersection, these same faces are present in the complex $T \cup D^{*} \cup \partial V \subset$ $V(0)$. $[\mathrm{P}]$ establishes torsion in the first homology of a subcomplex, a contradiction, since $V(0) \cong S^{3}$.

Suppose there are two Scharlemann cycles on $G_{D}$. Since $\alpha$ bounds at most one face of $G_{D}$ (recall $\alpha$ may be vacant), we can assume that at least one Scharlemann cycle is not incident to $\alpha$ and thus lies in the graph of intersection of $A$ and $D$. This is our contradiction, since a Scharlemann cycle above the sphere $A^{*}$ provides a lens-space summand in the ambient manifold, $V(\Delta) \cong S^{3}$.

Proof of Theorem 3.1. Taking $D$ itself to be our disk $C$ we apply Proposition 3.6 and Lemma 3.9.

Interestingly the techniques of [GL1] only narrowly fail in the proof of Theorem 3.1. For only one degenerate case, when $p=2$, do we require the stronger conclusion of Proposition 3.1 of [GL2], that in the absence of some type on $G_{T}$, we find two Scharlemann cycles on $G_{D}$. In [GL1], no assumption is made about the magnitude of $\Delta$. Consequently, those techniques guarantee only one Scharlemann cycle on $G_{D}$ if disk face representatives of all types cannot be found on $G_{T}$. If our geometrical interpretation relies on a Scharlemann cycle on $G_{D}$, we must insure that this cycle is not adjacent to $\alpha$, and indeed we can for $p>2$. Only when $p=2$ do we need two Scharlemann cycles. For this reason alone do we invoke the more powerful techniques of [GL2].

\section{On Composite Twisted Composite Knots}

In Appendix C, we produce a large infinite class of pairs of composite knots related by a single twist. Here we consider the circumstances under which the $(\Delta \geq 2, V)$-twisting of a composite knot $K=k \# k^{\prime}$ yields a composite knot. Clearly one obtains a composite $K_{\Delta}=k_{\Delta} \# k^{\prime}$ when a sphere splitting the summands of $K$ is contained within $V$. For the purposes of this analysis, if such a sphere exists, we say the $(\Delta, V)$-twisting of $K$ is a trivial twisting. Furthermore [HMo] gives an example of a pair of composite knots related through two full twists, the right and left handed granny knots. But no other pairs of composite knots can be related through twisting two or more full twists, as we set out to prove:

Theorem 4.1. If $K_{0}=K \subset V(0), K_{\Delta}=K \subset V(\Delta)$ are both composite knots then either $\Delta<2$ or $K_{\Delta}$ is obtained from $K_{0}$ by a trivial twisting, or $\Delta=2$ and $K_{0}, K_{\Delta}$ are the right and left handed granny knots. 
The unknot cannot arise as a trivial twisting of a composite knot; nor can any twisting of the granny knot give rise to an unknot by [MY] (or in this case, more directly, by examination since the proof precisely specifies the $(2, V)$-twisting relating the two granny knots); thus by Theorem 3.1, we have:

Theorem 4.2. At most one composite knot, either $K_{1}$ or $K_{-1}$, can arise from a particular $V$-twisting of an unknot $K$.

Assume, then, that $K_{0}, K_{\Delta}$ are in fact both composite, that $\Delta \geq 2$ and that $K_{\Delta}$ is obtained from $K_{0}$ by a non-trivial $(\Delta, V)$-twisting. We will examine the graphs of intersection of punctured spheres derived from the splitting spheres for $K_{0}$ and $K_{\Delta}$.

For distinct $\delta, \epsilon \in\{0, \Delta\}$, let $H_{\delta}$ be the core of the complement of $V$ in $V(\delta)$ and let $P_{\delta}^{*}$ be the sphere embedded in $V(\delta)$ that divides $K$ into knotted arcs, chosen so that $P_{\delta}$ intersects $H_{\delta}$ minimally and transversally. Let $P_{\delta}=P_{\delta}^{*} \cap V$. We can assume that $P_{0}, P_{\Delta}$ meet minimally. That is, all curves of intersection are arcs with ends on $\partial V$ or are simple closed curves essential in both $P_{\delta}-K$. Note further that no curves of intersection need meet $K$. Let $G_{\delta}$ be the graph on $P_{\delta}$ of the arcs of intersection with $P_{\epsilon}$ and $p_{\delta}$ be the number of boundary components of $P_{\delta}$. As $P_{\delta}$ separates, $p_{\delta}$ is even. Let $\hat{P}_{\delta}$ be the abstract two-sphere formed by contracting the the fat vertices of $G_{\delta}$ to points.

Note that if either $P_{\delta}^{*}$ does not meet $\partial V$, then $P_{\delta}^{*}$ would lie within $V$ and $K_{\Delta}$ would arise as a trivial twisting of $K_{0}$. Thus, each $P_{\delta}$ must have at least two boundary components, or $K_{\Delta}$ is obtained from $K_{0}$ by a trivial twisting.

As in previous sections, we can arrange that our graphs of intersection are as in [GL1] and [GL2]. All boundary components of $P_{\delta}$ are parallel and meet each boundary component of $P_{\epsilon}$ with multiplicity $\Delta$. Again the parity rule holds, for we are discussing the graphs of intersection of orientable surfaces in an orientable manifold.

By the minimality of the construction, we can assume any one-sided faces intersect $K$, since any one-sided faces missing $K$ could have been removed without affecting the intersections of the $P_{\delta}$ with $K$. There may be one or two one-sided faces on each $P_{\delta}$ which do meet $K$ (recall $K$ meets $P_{\delta}$ exactly twice). Such one-sided faces will not, in general, be removable unless we allow the number of intersections of $K$ with $P_{\epsilon}$ to increase. Consequently, we are forced to allow up to two such onesided faces in each $G_{\delta}$. In the combinatorics, such one-sided faces will be trivial Scharlemann cycles as well as trivially representing each type. These fail to produce torsion in the appropriate complex. Thus we attempt to apply the techniques of [GL2] to obtain enough Scharlemann cycles and representatives of each type to insure our result is of geometric use.

A trivial face of $G_{\delta}(L)$ is a disk face meeting $K$. Note there are at most two such faces on each $P_{\delta}$. A non-trivial representative on $G_{\delta}(L)$ of an $L$-type $\tau$ will be any disk face representing $\tau$ that is not trivial. Not only does this definition allow us to avoid choosing one-sided faces as representatives, but in the final proof insures that any disk face representing a type does not contain any simple closed curves of intersection (any such curve, recall, would be essential in $P_{\delta}-K$ ). Similarly we ask that a non-trivial Scharlemann cycle be a Scharlemann cycle missing $K$.

We must be particularly careful with our induction to avoid producing a trivial Scharlemann cycle. We can eliminate one trivial face by begining our induction in 
a regular neighborhood of the complement of this face. We try to avoid working our way towards the other trivial face.

We define a non-trivial $x$-edge cycle, relative to a region $C$, as an $x$-edge cycle bounding a disk $C_{0} \subset C$ such that $C_{0}$ does not contain a trivial face. The interior vertices of an $x$-edge cycle are those in the interior of $C_{0}$. Define a trivial $x$-web as an $x$-web having only two components in its complement. (Note that a trivial $x$-web consists of exactly one vertex and one loop edge when $\Delta \geq 2$.) A non-trivial $x$-web provides an interior component not containing a trivial face, i.e. a non-trivial $x$ edge cycle. We induct within this, ultimately producing a non-trivial Scharlemann cycle. Figure 18 illustrates these $x$-objects. Note that all vertices of any $x$-object are of the same parity.
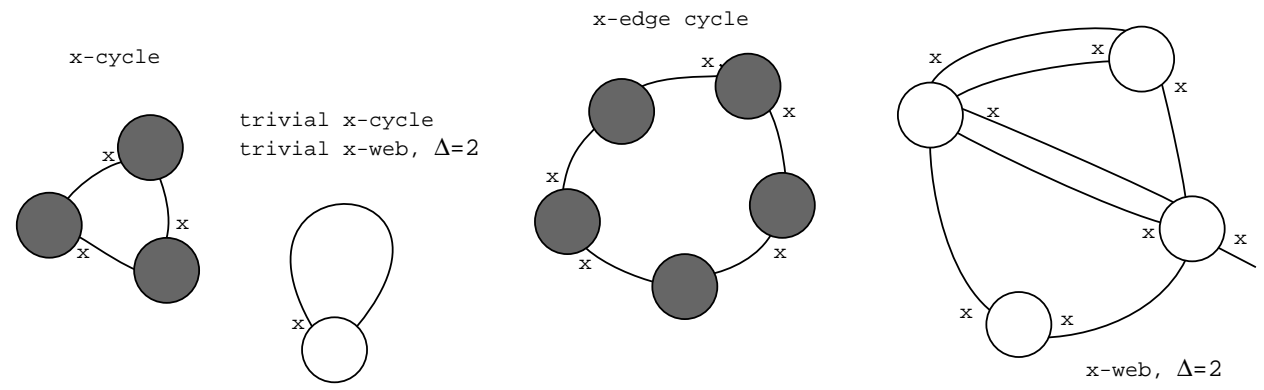

Figure 18. Various $x$-objects

As the following lemma points out, when $\Delta=2$, we may not be able to avoid the trivial $x$-objects; a certain class of type may have only two (possibly trivial) representatives, yet will provide only trivial $x$-webs with which to induct. In turn this induction may steadily hone in on a trivial Scharlemann cycle. In this case we instead use certain structures arising from a special case outlined below to fully describe the graph. Roughly speaking, the two cases in the conclusion of the lemma below correspond to the second two cases in the proof of Proposition 4.6.

Lemma 4.3. Take $P_{0}, P_{\Delta}$ as above. Let $L$ be a set of labels in $G_{0}$. For a particular $L$-type $\tau,|L| \geq 2$, with all elements of $C(t)$, or all $A(t)$ having the same parity, take $t$ to be a star representing $\tau$ and $L(t)=L$. Let $c=|C(t)|=|A(t)|$. If $\Delta \geq 2$, then there is a non-trivial representative of $\tau$ in $G_{0}(L)$ or

(i) if $\Delta c \geq 3$ then there is a non-trivial $x$-web $\Sigma$ in $G_{\Delta}$ such that all vertices of $\Sigma$ are in $C(t)$ or all are in $A(t)$.

(ii) if $\Delta=2$ and $c=1$ then there are $p_{0}$ loop edges incident to the two vertices of $G_{\Delta}$ in $C(t) \cup A(t)$.

Proof. Let $r$ be the number of disk face representatives of $\tau$ and $s$ be the number of switch edges in $G_{0}(L)$. As in Lemma 2.3.2 of [GL1], with the changes noted in the proof of Lemma 3.3, a simple Euler characteristic count reveals that $r+s \geq$ $(\Delta c-1) p_{0}+\chi\left(\hat{P}_{0}\right)$. If $r \geq 3$ there is a non-trivial representative of $\tau$. Assume then that $r \leq 2$. Then there are at least $(\Delta c-1) p_{0}$ switch edges in $G_{0}$. Thus at least $(\Delta c-1) p_{0}$ of the $\Delta c p_{0}$ occurrences of the labels in, say, $C(t)$ are incident to switch edges. There is then some vertex $v$ of $G_{0}$ such that at least $(\Delta c-1)$ switch edges are each incident to exactly one of the $\Delta c$ occurrences of the labels of $C(t)$ at $v$. (Note that the switch edges cannot be loop edges by the parity rule). 
Then on $G_{\Delta}$ we see $(\Delta c-1) v$-edges connecting parallel vertices in $C(t)$. Let $\Lambda$ be the subgraph of the vertices of $C(t)$ in $G_{\Delta}$ and these $v$-edges. Note that any component of $\Lambda$ will be a $v$-web. We take $\Sigma$ to be any component of $\Lambda$ with more edges than vertices. In the first case of the lemma, $\Delta c \geq 3$ and thus such a component must exist. Then $P_{0}-\Sigma$ has at least three components and $\Sigma$ is non-trivial.

In the second case of the lemma, simply note that if there are no non-trivial representatives of $\tau$ then there are at least $p_{0}$ switch edges. Such edges have both ends incident to either the single clockwise switch label or the single anticlockwise switch label. On $G_{\Delta}$ we see $p_{0}$ loop edges incident to the vertices corresponding to these labels.

4.1. The Special Case. Hayashi and Motegi [HMo] give an example of a pair of composite knots related through a $(2, V)$-twisting, the right and left handed granny knots. In order to establish our approach to the proof of Theorem 4.1, we must first examine the special case that leads to trouble with the usual techniques. We will see that the sorts of graph of the special case are exactly those for which the second case of Lemma 4.3 must be invoked; that is, these graphs lack representatives of some loop $(L)$-type, an $(L)$-type $\tau$ such that there exists a $\tau_{k}=d_{k}(\tau)$ such that $\tau_{k}$ has only one clockwise switch.

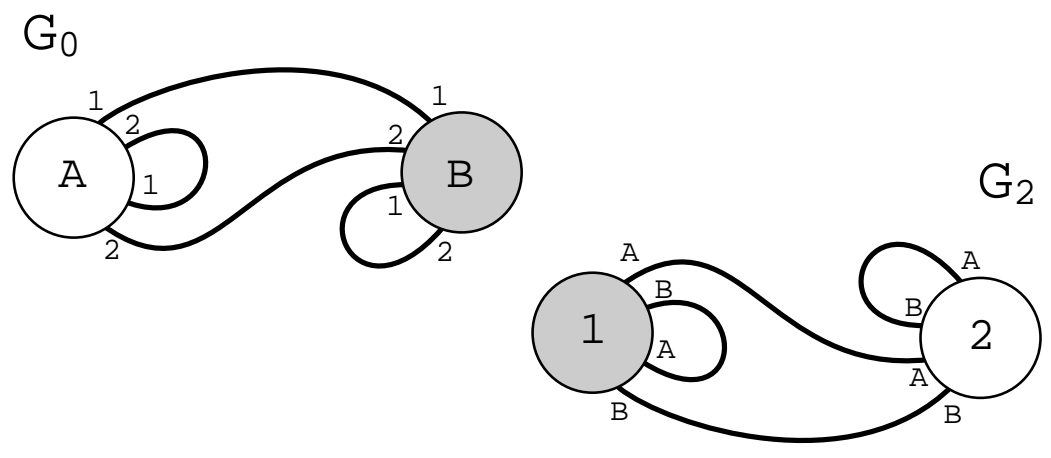

FiguRE 19. A pair of graphs which induce the granny knot

The graphs which lead to the special case, and the complex they induce, are illustrated in Figures 19 and 20. This is the only pair of two-verticed graphs $G_{0}$ and $G_{2}$ that could possibly arise from two composite knots by a non-trivial $(2, V)$ twisting. In Lemma 4.4 we show this pair of graphs must induce a granny knot in each $V(\delta)$. In Lemma 4.5 we note that any pair of ring graphs $G_{0}, G_{2}$ arising from a non-trivial $(2, V)$-twisting can be replaced by the graphs of Figure 19. Finally, in Proposition 4.6, we show that any pair of graphs of intersection that could have actually arisen from a non-trivial $(\Delta \geq 2, V)$-twisting must in fact be ring graphs with $\Delta=2$, completing the proof of Theorem 4.1.

Lemma 4.4. Suppose two-verticed $G_{0}, G_{2}$ arise from a non-trivial $(2, V)$-twisting relating composite knots $K_{0}=K \subset V \subset V(0)$ and $K_{2}=K \subset V \subset V(2)$. Then the composite knots $K_{0}$ and $K_{2}$ must be granny knots of opposite parity. 


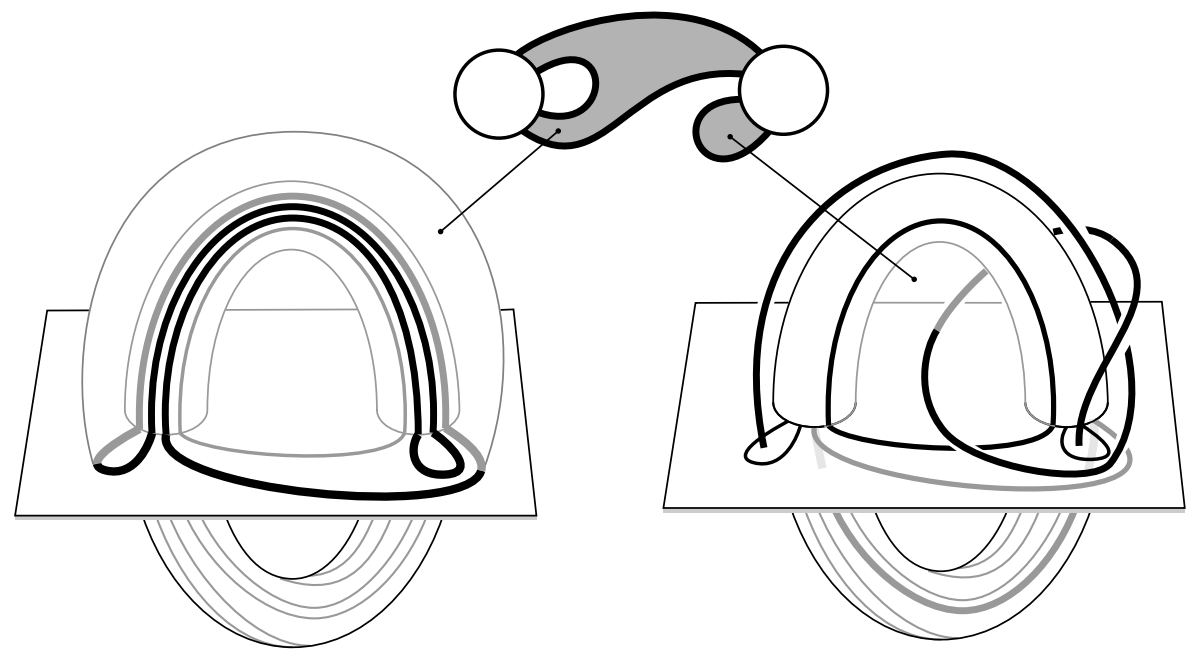

FiguRE 20. The granny knot results from the graphs

Proof. A quick analysis shows the graphs of intersection must be as in Figure 19, or contain Scharlemann cycles, or have more than two one-sided faces, or have the obstruction described in the second paragraph of the proof of Lemma 4.5.

Consider the complex in, say, $V(2)$. The one-sided faces of $G_{0}$ lie on opposite sides of $P_{2}$ and must be incident to different edges of $G_{2}$ connecting the fat vertices of $G_{2}$. Note that the one-sided faces fix the position of the handles (the components of $S^{3}-V-P_{2}^{*}$ ) with respect to the graph $G_{2}$, up to an orientation reversing homeomorphism, and so the placement of the trigonal faces is forced. Thus the complex in $V(2)$ is as pictured in Figure 20.

We now note that any knot $K \subset V \subset V(2)$, passing through each one-sided face of $G_{2}$ and $G_{0}$ exactly once and missing the complex $P_{0} \cup P_{2} \cup \partial V$ elsewhere, must in fact be a granny knot.

In Figure 20 the shaded faces of $G_{0}$ lie to one side of $P_{2}$, forming a trigonal disk pictured at left and a one-sided disk pictured at right. We will see these disks force a trefoil summand $k$ of our composite knot $K$ in $V(2)$. Above $P_{2}$ the boundary of a neighborhood of the shaded trigonal face of $G_{0}$, a portion of $\partial V$, and $P_{2}$ itself together bound a ball $B$ containing a summand $k$ of $K$ and contained within $V$. The $K_{\delta}$ are related by a non-trivial twisting and so $k$ must be unknotted within $B$. Thus $k$ must be as pictured in the right of Figure 20, a trefoil summand. Similarly we see that the cosummand for $k$ must be a trefoil of the same parity, and so $K$ is a granny knot in $V(2)$, meeting each one-sided face of the complex exactly once. Since the twisting operation preserves the orientation of $V$, and thus sends the $(2,1)$ curves of $\partial P_{0} \subset \partial V$ to $(0,1)$ curves and the $(0,1)$ curves of $\partial P_{2} \subset \partial V$ to $-(2,1)$ curves, we see that in $V(0)$, the picture of the complex is exactly reversed, and $K$ is thus a granny knot of the opposite parity in $V(0)$. For later use, note $k$ can be isotoped to actually lie on the boundary of a neighborhood of the complex. 
We now analyze the class of graphs leading to trouble with the usual techniques. We show that they could only lead to the conditions required for the application of Lemma 4.4, and thus to the example of [HMo]. Assume $\Delta=2$ for this discussion.

$G_{\delta}$ is a ring graph if it consists of nested ring annuli. A ring annulus is an annulus in $\hat{P}_{\delta}$ containing exactly two vertices of opposite parity connected by exactly $p_{\epsilon}$ edges, and at each vertex the remaining labels are incident to concentric loop edges parallel to the boundary of the annulus. Note that if each of the $G_{\delta}$ is a ring graph, then all edges incident to two different fat vertices have both ends on the same label. Note also that our ring graphs have exactly two one-sided faces, and thus in either $\hat{P}_{\delta}$, all loop edges are concentric. Finally, the faces of a ring annulus in $\hat{P}_{\delta}$ must be: two punctured one-sided faces incident to the boundary of the annulus, and either $2 p_{\epsilon}-4$ bigons and two trigons, or $2 p_{\epsilon}-3$ bigons and a quadrilateral. A pair of ring graphs, with their labels removed for clarity, is illustrated in Figure 21.

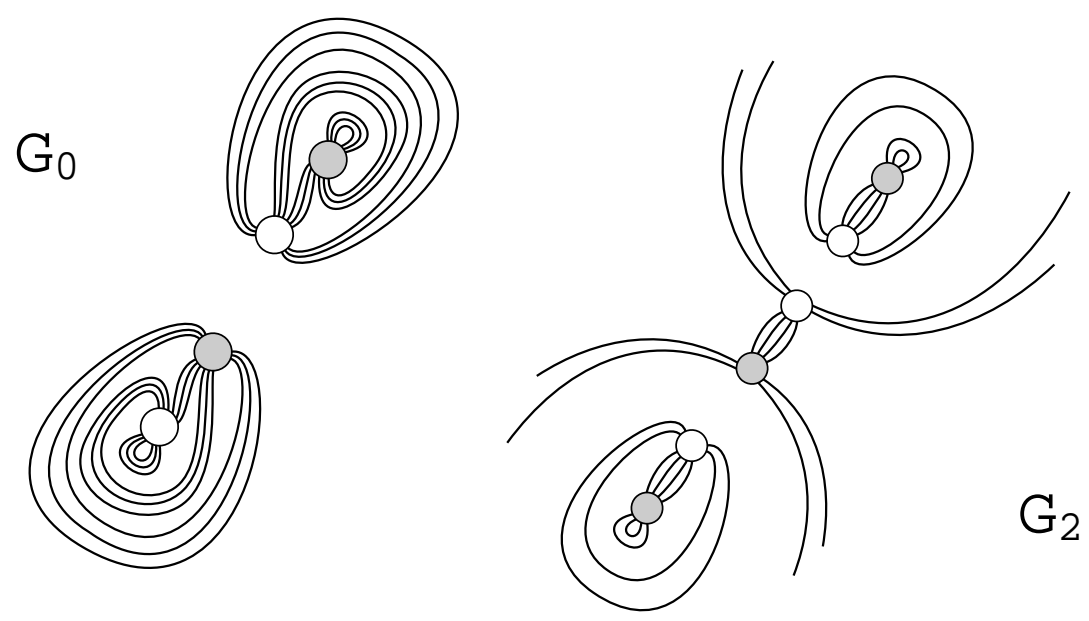

Figure 21. A pair of ring graphs

Lemma 4.5. Let $V$ be a solid torus. Suppose $G_{0}, G_{2}$ are ring graphs arising from a pair of composite knots $K_{0}=K \subset V \subset V(0)$ and $K_{2}=K \subset V \subset V(2)$ related through a non-trivial $(2, V)$-twisting. Then we can find splitting spheres for $K_{0}$ and $K_{2}$ that each meet $\partial V$ twice.

Proof. We show that $K_{2}$ has such a splitting sphere. The argument for $K_{0}$ is exactly the same. We analyze such graphs in a solid torus directly: Let the innermost ring annulus have fat vertices $x, y$ on $G_{0}$, say, and be incident to a one-sided face $C$ on labels, say 1 and 2. Then we consider two cases: in this annulus, there is a bigon $C_{1}$ between $x$ and $y$ with ends incident to the labels 1 and 2, or there is not and thus a trigon $C_{2}$ is incident to the remaining occurrences of the labels 1 and 2. Note that the first case arises exactly when there is a quadrilateral in the annulus.

Consider the first of these cases. Above $P_{2}$ we examine the structure formed by $C$ and $C_{1}$ and the segment $S$ of the boundary of $V$ running between vertices 1 and 2 of $G_{2}$ and vertices $x$ and $y$ of $G_{0}$. Clearly $C_{1}$ and $S$ form an annulus above $G_{2}$. Then $C$ lies to one side of this annulus and the one-sided faces of $G_{2}$ to the other, since all loop edges are parallel. $K$ must meet $C$, cannot meet $G_{2}$ to the same side 
of the constructed annulus as $C$, and yet cannot escape to meet $G_{2}$ elsewhere. But of course, $K$ must meet $G_{2}$ twice, by construction, and we have a contradiction (Figure 22).

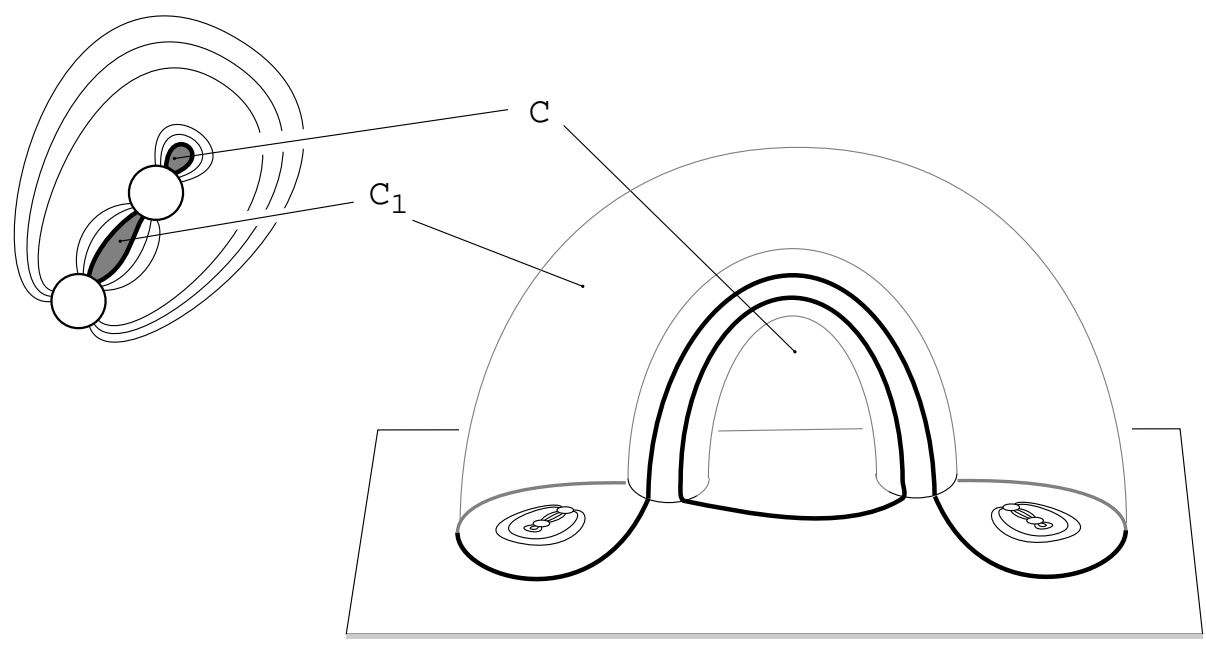

Figure 22. There are no bigons on the same label pair as a onesided face

On the other hand, suppose a trigon $C_{2}$ is incident to the remaining occurrences of the labels 1 and 2 in the ring annulus incident to $C$. We will produce a splitting sphere for $K$ in $V(2)$ that meets $\partial V$ exactly twice. In $V(2)$, let $S$ be the portion of the complement of $V$ between the fat vertices 1 and 2 that lies to the same side of $P_{2}$ as $C_{2}$ and $C$, and $R$ be the ring annulus incident to fat vertices 1 and 2 . Note that $C$ trivializes the handle $S$ and thereby fixes the relative positions of $C_{2}$ and $R$ (Figure 23). Let $B$ be a small neighborhood of $R \cup S \cup C \cup C_{2}$. Note that $\partial B$ meets $\partial V$ exactly twice, and that because $K$ must meet $C$, exactly one arc $k$ of $K$ lies within $B$. Furthermore, $B$ is a ball, as can be seen by noting how the neighborhood of the trigonal face can be isotoped to close off the hole at the boundary of the neighborhood of the ring annulus $\left(C_{2}\right.$ can slide over $\left.C\right)$. Finally, we note that there is no isotopy of $k$ to the boundary of $B$ holding the ends of $k$ fixed, because the union of $k$ with an arc on the boundary of $B$ connecting the ends of $k$ is a trefoil knot. Such an arc can be produced by considering the trefoil summand from the discussion of Figure 20. Recall that this summand could be isotoped, in effect, to the boundary of $B-N(C)$.

Since $(B, k)$ can be gently isotoped to lie above $P_{2}$, the complement of $B$ in $V(2)$ also contains a single, knotted arc of $K$ and so $\partial B$ is a splitting sphere for $K$ that meets $\partial V$ exactly twice.

So we know that there are only two fat vertices on both of the $P_{\delta}$. Consequently, the graphs $G_{\delta}$ must be as in Figure 19, and so if our composite knots $K_{0}$ and $K_{2}$ are related by a non-trivial twist, they must be the right and left handed granny knots by Lemma 4.4. 


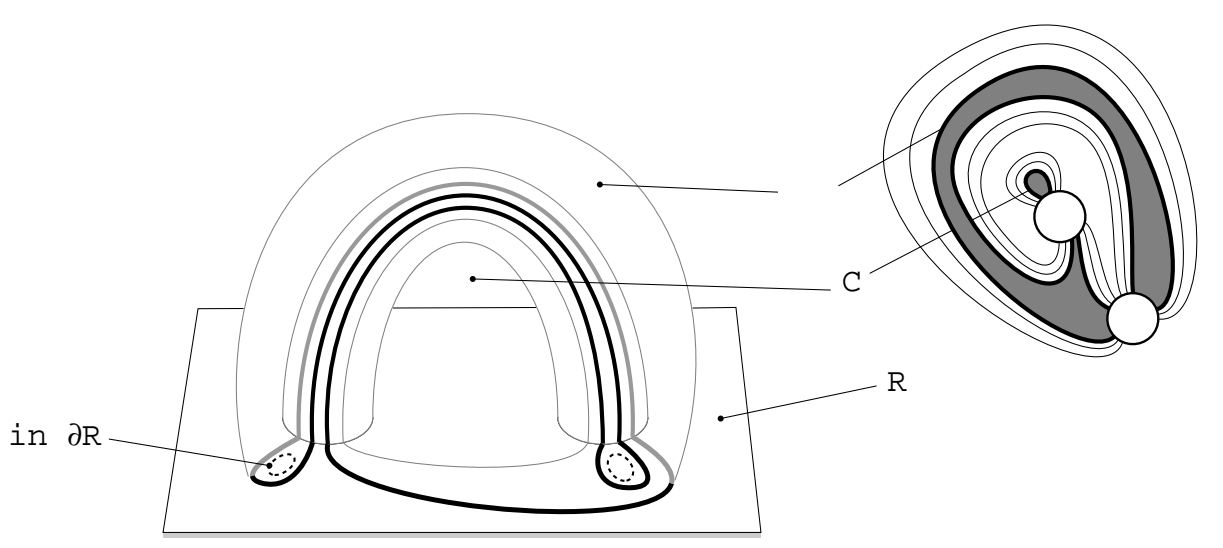

Figure 23. $R \cup S \cup C \cup C_{2}$

In Section 4.2 we aim to show that any pair of graphs $P_{0}, P_{\Delta}$ that give rise to a complex that can embed in $S^{3}$ is in fact a pair of ring graphs. To do this we define a loop annulus $A \subset \hat{P_{\Delta}}$ to be an annulus such that:

(i) all edges incident to vertices of $G_{\Delta}$ in $A$ are contained within $A$;

(ii) $A$ contains no trivial faces;

(iii) either there is an external vertex, $v$, a vertex incident to at least $p_{0} / 2$ loop edges that are parallel in $P_{\Delta}$ to the external boundary of $A$;

or $A=\hat{P_{\Delta}}-\left\{x_{1}, x_{2}\right\}$ where the $x_{i}$ are each points in different faces of $G_{\Delta}$ and there is no external vertex.

Although a loop annulus' exterior vertex may or may not lie within the annulus itself, by construction we will see that the exterior vertex always lies within some loop annulus. The interior vertices of $A$ are the vertices of $G_{\Delta} \cap A-x$ where $x$ is the external vertex. See Figure 24.

4.2. The General Case. The following is a reworking of Proposition 3.1 of [GL2]. In order to compensate for the presence of trivial faces on $G_{\Delta}$, we must take extra measures to control the course of our induction.

Proposition 4.6. Let $P_{\Delta}, P_{0}$ be as above. Let $\Delta \geq 2$. Let $C \subset \hat{P_{\Delta}}$ contain no trivial faces and be one of

(i) a disk bounded by a non-trivial x-edge cycle, $\Sigma$;

(ii) a disk with interior vertices and boundary $\Sigma$ a collection of loop edges all incident to a single vertex or

(iii) a loop annulus, with $\Sigma=\emptyset$.

Let $L$ be the interior vertices of $C$. Then either $G_{\Delta}$ or $G_{0}$ contains a non-trivial Scharlemann cycle, each $L$-type $\tau$ has a non-trivial representative on $G_{0}(L)$, or $C$ can be partitioned into ring annuli.

To accomplish this, we seek a Scharlemann cycle within $C$ on $G_{\Delta}$, or three Scharlemann cycles on $G_{0}$, or a non-trivial representative of each $L$-type on $G_{0}(L)$. In cases (i) and (ii), this will succeed. However if $C$ is a loop annulus, we face the additional possibility that we lack non-trivial representatives of loop types. In this case, we inductively show the loop annulus is a ring annulus or can be partitioned 
into loop annuli with fewer internal vertices or contains a disk bounded by a nontrivial x-cycle..

We will proceed by induction on $|L|$. Thus we begin with:

Lemma 4.7. Taking the hypotheses of Proposition 4.6, $|L|>1$, or $C$ is a ring annulus, or one of the $G_{\delta}$ contains a non-trivial Scharlemann cycle.

Proof. If $C$ is a loop annulus with $L=1$, then clearly $C$ is a ring annulus or contains a Scharlemann cycle. Thus assume $C$ is a disk. The proof is now the same as Lemma 3.2 of [GL2], noting that $\Lambda$ is a non-trivial great $v$-web and thus contains a non-trivial Scharlemann cycle.

Proof of Proposition 4.6. This is modeled on the proof of Proposition 3.1 of [GL2], using Lemma 4.7 for Lemma 3.2 of [GL2] and Lemma 4.3 for Lemma 2.5 of [GL2]. We assume $|L| \geq 2$.

In the first two cases of the hypothesis, we proceed much as in the proof of Proposition 3.1 of [GL2], with relatively minor changes.

In the third case of the hypothesis, we find all $L$-types on $G_{0}$, or obtain a disk $C_{0} \subset C$ in which we can induct, or if a loop $L$-type is missing under specific circumstances, we partition the loop annulus into smaller loop annuli, with fewer internal vertices and whose external vertices correspond to the switches of the missing looptype's $\tau_{k}$. The lack of a non-trivial non-loop type yields a non-trivial Scharlemann cycle or one of the structures given in the first two cases of the hypothesis of the proposition.

We group our arguments by $L$-type (the trivial $L$-type is defined in [GL1]):

(1) $\tau$ is the trivial $L$-type.

(2) $\tau$ is non-trivial and not a loop type.

(3) $\tau$ is a loop-type.

In general, we note that if at any point we find a non-trivial [great] $x$-web within $C$ we can find a non-trivial [great] $x$-edge cycle $\Sigma_{0}$, a subset of this $x$-web, bounding a disk $C_{0} \subset C$. Also, by Lemma 2.2 of [GL2], note that if $C$ is bounded by a nontrivial $x$-edge cycle and the interior of $C$ contains no vertices of $G_{\Delta}$ then $C$ contains a non-trivial Scharlemann cycle.

(1) $\tau$ is trivial. We follow the proof of Case (1) of Proposition 3.1 of [GL2]. If $C$ is a disk, let $J$ be the set of interior vertices of $C$ of opposite parity of the vertices of $\Sigma$; or if $C$ is a loop annulus, let $J$ be the set of interior vertices of opposite parity of any external vertex lying in the interior of $C$. (If $\Sigma$ is vacant or if $C$ is a loop annulus without an interior external vertex, choose the $J$ to be the larger class of parallel vertices interior to $C$.) If $J$ is vacant then there is a non-trivial great $x$-edge cycle bounding a disk in $C$, and consequently a non-trivial Scharlemann cycle in $G_{\Delta}$.

Thus assume $|J| \geq 1$. If for each vertex $y$ of $G_{0}$ there are two distinct edges, each meeting $y$ at labels in $J$ and connecting $y$ to parallel vertices, we proceed as in [GL2] and obtain at least four representatives of the trivial $L$-type on $G_{0}$. Otherwise, we find ourselves in Case (1.b) of the proof in [GL2] and obtain $(\Delta|J|-1) x$-edges incident to the vertices of $J$. Thus if $C$ is a disk, or if $|J| \geq 2$, or if $\Delta \geq 3$, we are guaranteed a non-trivial $x$-edge cycle $\Sigma_{0}$ incident to the vertices of $J$, bounding a disk $C_{0}$. In this case, we let $L_{0}$ be the vertices of $G_{\Delta}$ within $C_{0}$ and follow the last half of Case (1.b) of Proposition 3.1 of [GL2]. 
So assume that $C$ is a loop annulus, that we must have chosen $J$ to contain only a single vertex $v$, and that $\Delta=2$. Let $I$ be the set of vertices of $C$ not in $J$ and suppose $|I|=1$; this vertex must have parity opposite to the parity of $v$. Now if there are more than $p_{0} / 2$ loop edges incident to $v$, we find a non-trivial Scharlemann cycle among these edges; if there are exactly $p_{0} / 2$ loop edges incident to $v$, then $C$ is a ring annulus. If there are fewer than $p_{0} / 2$ loop edges incident to $v$, then there are at least $p_{0}+1 v$-edges on $G_{0}$ with ends on parallel vertices; consequently there are at least three disks on $P_{0}$ bounded by such edges. These disks are in fact bounded by a great $v$-edge cycle and so each contains a Scharlemann cycle on $G_{0}$, one of which must be non-trivial. Then if $C$ contains only one other vertex besides $v$, we have proven the proposition.

Assume then that $|I| \geq 2$. Since $(\Delta|J|-1) \geq 1$ there is at least one loop edge incident to the vertex in $J$, and thus there is a label $w$ such that at most one of the $2|I|$ occurrences of $w$ on the vertices of $I$ is incident to an edge meeting $v$. But since the vertices of $I$ are all parallel, the parity rule insures that there are at least $2|I|-1$ distinct $w$-edges with both ends on vertices of $I$. A quick Euler characteristic count yields at least one disk $C_{0}$ in $C$ bounded by an $w$-edge cycle $\Sigma_{0}$. In this final case, then, we let $L_{0}$ be the vertices of $G_{\Delta}$ within $C_{0}$ and follow the last half of Case (1.b) of Proposition 3.1 of [GL2].

(2) $\tau$ is non-trivial and not a loop-type. This almost exactly follows Case (2) of Proposition 3.1. However, there is one important change in the actual inductive step. If we fail to find the requisite non-trivial representative of a particular $L$-type $\tau$, we proceed as follows (refer to the top of page 18, [GL2], applying Lemma 4.3 in place of Theorem 2.5 of [GL2]):

We find a non-trivial $x$-web $\Sigma^{\prime}$ within $C$. We can still assume that the vertices of $\Sigma^{\prime}$ are contained within $C(t)$ by the reasoning in [GL2]. Let $\Sigma_{0} \subset \Sigma^{\prime}$ be a nontrivial $x$-edge cycle bounding a disk $C_{0}$ in $C$ and take $L_{0}$ to be the vertices of $L$ in $C_{0}$. Note $\left|L_{0}\right|<|L|$ and we can thus proceed with the induction as given in the last paragraph of the proof of Case 2 of Proposition 3.1 of [GL2].

(3) $\tau$ is a loop type. If $\Delta \geq 3$ then we apply the argument of the case above. If $\Delta=2$, and $C$ is not a loop annulus then applying Lemma 4.3, we produce two loop vertices within $C$ together incident to at least $p_{0}$ loop edges. One of these vertices $v$ then is incident to at least $p_{0} / 2$ loop edges. After a possible change of orientation, as in [GL2], we can assume $v \in C(t)$. If there is a disk $C_{0} \subset C$ containing interior vertices bounded by loop edges incident to $v$, we take $\Sigma_{0}$ to be the bounding edges of $C$ and $L_{0}$ to be the interior vertices of $C_{0}$. We then proceed exactly as in the second paragraph of the proof of Case 2 of of Proposition 3.1 of [GL2]. If there is no such disk, then an innermost loop edge incident to $v$ bounds a one-sided face, a contradiction.

We now consider the motivating case: $\tau$ is a loop type, $\Delta=2$, and $C$ is a loop annulus. We set forth as in Case 2 of Proposition 3.1 of [GL2], constructing a sequence of stars, etc. until reaching $\tau_{k}=d_{k}(\tau)$, such that $\left|C\left(t_{k}\right)\right|=1$. If there is a non-trivial representive of $\tau_{k}$ in $G_{0}\left(L_{k}\right)$ then we are done just as in [GL2]. Otherwise the two vertices $v_{1}, v_{2}$ of $C\left(t_{k}\right) \cup A\left(t_{k}\right)$ are incident to at least $p_{0}$ loop edges. We seek either a Scharlemann cycle in $C$, or a disk $C_{0} \subset C$ bounded by loop edges incident to one of the $v_{i}$ in which to induct, or to partition $C$ into loop annuli with fewer internal vertices. We have three cases:

If one of these vertices is incident to more than $p_{0} / 2$ loop edges we find a nontrivial $x$-edge cycle $\Sigma_{0}$ bounding a disk $C_{0} \subset C$. 

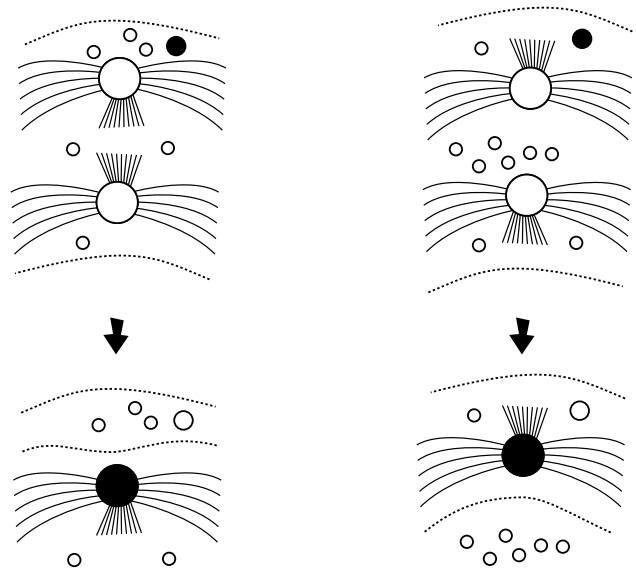

o
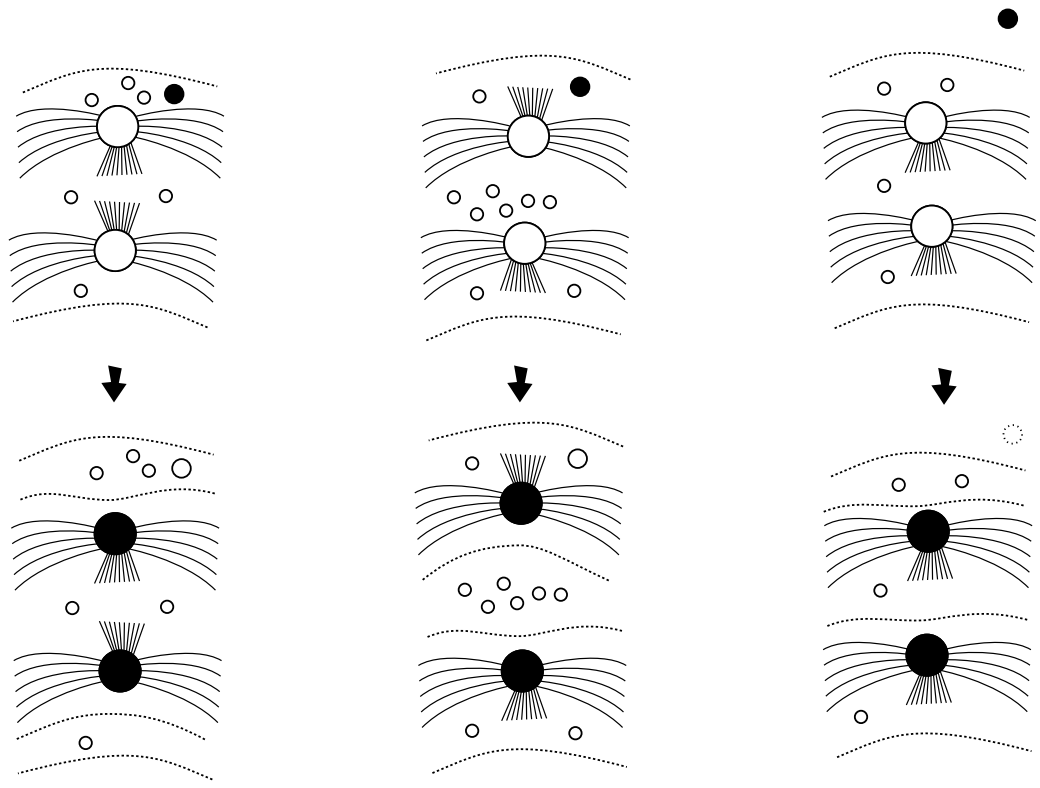

Figure 24. Partitioning loop annuli

Thus assume both vertices are incident to exactly $p_{0} / 2$ loop edges. If these edges are not all concentric in $\hat{P_{\Delta}}$ with the boundary of $C$, or if one of these vertices has loop edges that are not parallel in $P_{\Delta}$, we find a a disk $C_{0} \subset C$ bounded by a collection of loop edges $\Sigma_{0}$, all incident to a single vertex, but containing at least one interior vertex.

In either of these situations, we again proceed as in the final paragraph of Case 2 of Proposition 3.1 of [GL2].

Finally we consider the case when the $p_{0} / 2$ loops incident to $v_{i}$ are parallel in $P_{\Delta}$ for each $v_{i}$. Note that at either $v_{i}$ the loops must all be incident to a single label interval, or there would be some label $x$ such that two parallel $x$-edges would be incident to one of the $v_{i}$. These would bound a great $x$-cycle which would contain a non-trivial Scharlemann cycle.

Partition the original loop annulus $A$ along curves parallel to the loop edges incident to the $v_{i}$ (see Figure 24). We thus get three smaller loop annuli. First, note that for each new loop annulus, at least one of the $v_{i}$ can serve as an external vertex. If both do, choose either. Second, one of the new annuli may be vacant. Simply ignore any such annulus. If the original loop annulus contained its external vertex, this vertex will be internal to one of the new annuli. However, there are fewer internal vertices among all the new loop annuli together than there were in the old. (Note that if the outer annuli are vacant in the case on the left of Figure 24 then the original loop annulus did not contain its external vertex, and thus we have one less internal vertex than before.)

Thus the induction proceeds. Either each of the new loop annuli are ring annuli, or one of the new loop annuli, say $A_{0}$, containing interior vertices $L_{0}$ contains a non- 
trivial representative of each $L_{0}$-type. As before, we may assume that the external vertex of $A_{0}$ is the clockwise switch of $d_{k}(\tau)$. We proceed as at the end of Case 2 of Proposition 3.1 of [GL2]. The external vertices are black in the illustration; the internal vertices are white.

Thus we can either find our loop $L$-types or, by induction on $|L|$, we see that $C$ is composed of ring annuli.

Proof of Theorem 4.1. Suppose there are $k \in\{0,1,2\}$ trivial faces in $G_{\Delta}$. Choose $\mathrm{k}$ points from the distinct interiors of these faces, $2-k$ points from any components of $P_{\Delta}-G_{\Delta}$, and let $A$ be $\hat{P_{\Delta}}$ with these points removed. Thus $A$ contains no trivial faces.

Applying Proposition 4.6 we have, by the techniques mentioned at the end of Section 3, torsion in either $V(\Delta)$ or $V(0)$, a contradiction, or $G_{\Delta=2}$ was a ring graph. By switching our choice of which composite knot is $K_{0}$ and which is $K_{\Delta}$ we again obtain a contradiction or $G_{0}$ is a ring graph. Thus, by Section 4.1 , we have in this case that our composite knots related through twisting are the right and left handed granny knots.

The loop type techniques should be more widely applicable to settings on surfaces with genus, where there are at least a few essential curves on the surface, missing the graphs of intersection.

\section{Appendix A. Infinite FAmilies of GRAPhs With $|\partial A|>2$}

All published examples, and all the examples classified in Section 2, arise when the splitting sphere for $K_{1}$ is punctured twice by $\partial V$, that is, when $A$ divides $V$ into two solid tori, each containing a component of $K_{1}$, as pictured at left, below.

For the moment one must wonder if examples for which $\partial V$ must puncture the splitting sphere more than twice can even arise. If such an example exists, the punctured spheres would appear as at the right in Figure 25, two or more annuli tubed together.
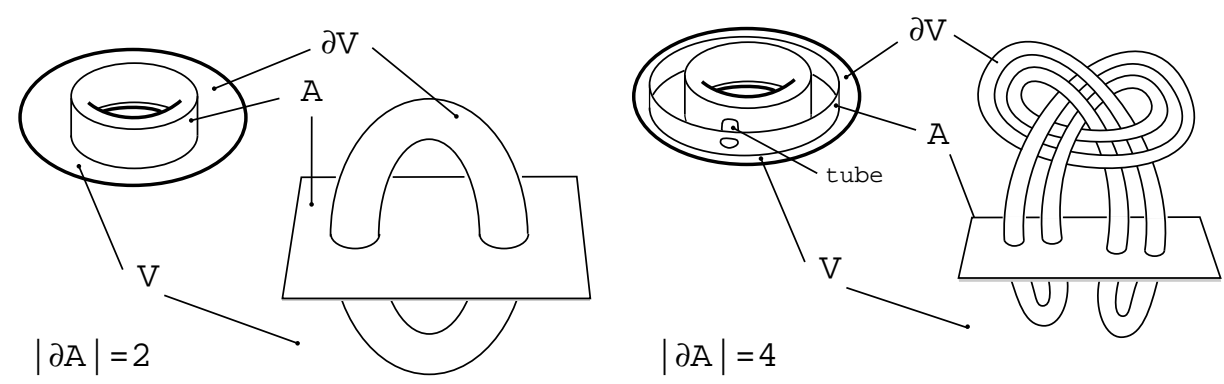

Figure 25. Possible splitting spheres in $V$

Interestingly, there are graphs of intersection that might serve as potential $G_{A}$ and $G_{D}$, such that $G_{A}$ has more than four boundary components on $\partial V$, and such that neither graph has all types or Scharlemann cycles. However, for the time being, all known examples of these graphs have more subtle obstructions, either to properly embedding the induced complex $A \cup D$ in a solid torus $V$, or to producing two non-trivial summands for the twisted unknot $K=\partial D \subset V(1)$. For example, 
the pair of graphs in Figure 26 cannot be correctly embedded in a solid torus, but the torsion in the complex cannot be detected by the combinatorics developed in [CGLS], [GL1] and [GL2]. The pair of graphs in Figure 27 contains an abundance of parallel klein bottles. The pair of graphs in Figure 28 can be embedded correctly in a solid torus, but both of the summands of $K$ must be trivial. The pairs of graph in Figures 27 and 28 can each be extended to an infinite family of pairs of graphs.
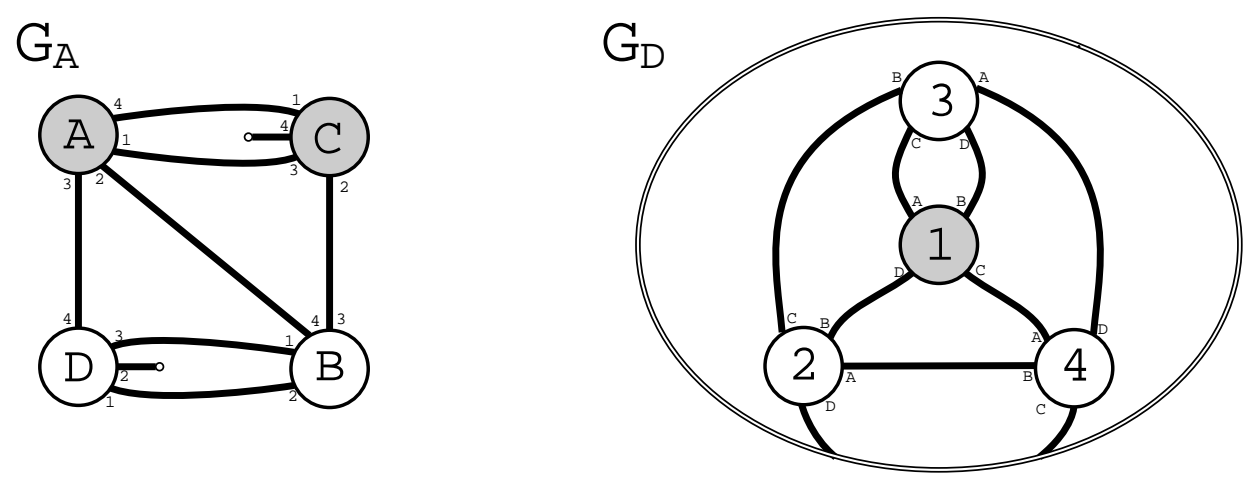

Figure 26. A pair of graphs of intersection with torsion but not all types
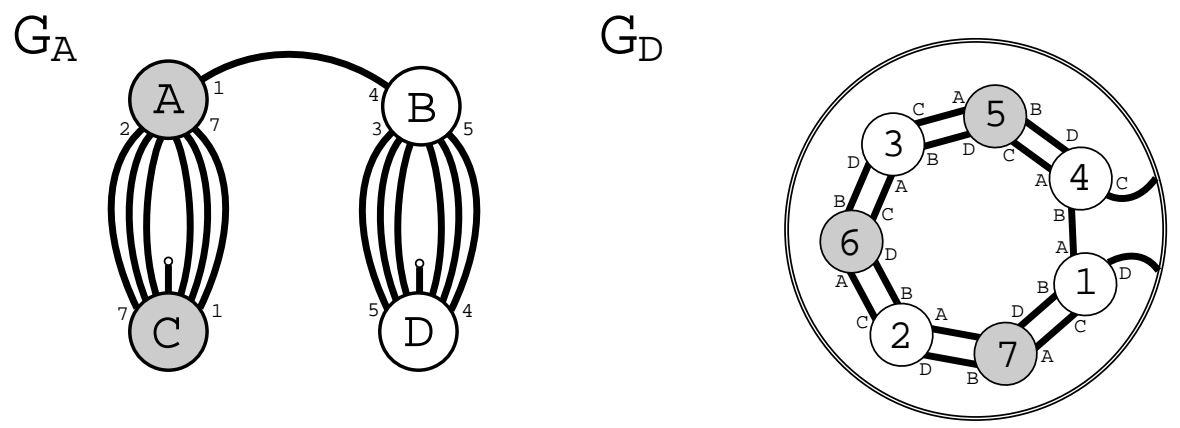

FiguRE 27. A pair of graphs containing klein bottles

\section{Appendix B. Presentations of Published examples of COMPOSITE TWISTED UNKNOTS}

The first example below is from [Mo]. The third and fifth are from [MY]. In all the examples, the twists in $G_{A}$ are normalized to fit parallel $(1,1)$ curves on $\partial V$, just as $G_{A}$ of Figure 9 is related to the arcs on $A \cup \partial V \subset V$ in Figure 7 .

1. Our first example, $(3,2) \#(5,2)$ was published in [Mo] (Figure 29).

2. $(3,2) \#(5,-2)$, shown in Figure 30 generalizes to the twisted unknot $(p, q) \#$ $(p+q,-q)$ for all relatively prime $p, q$. Here $G_{A}$ is parametrized by $a, b$, and $j$ : if 

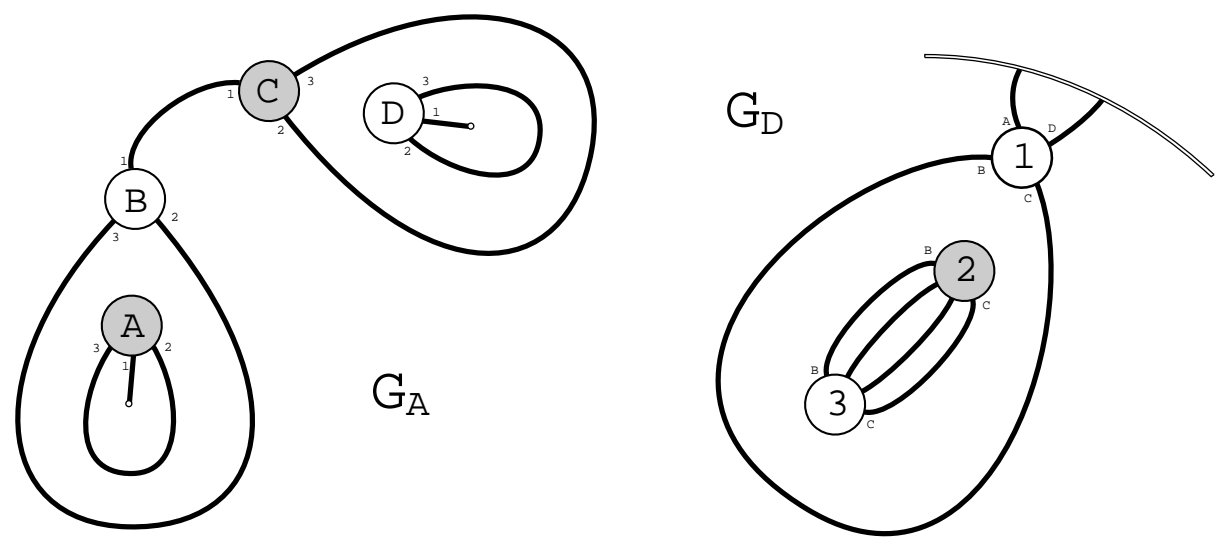

Figure 28. A pair of graphs that induces only an unknotted twisted unknot

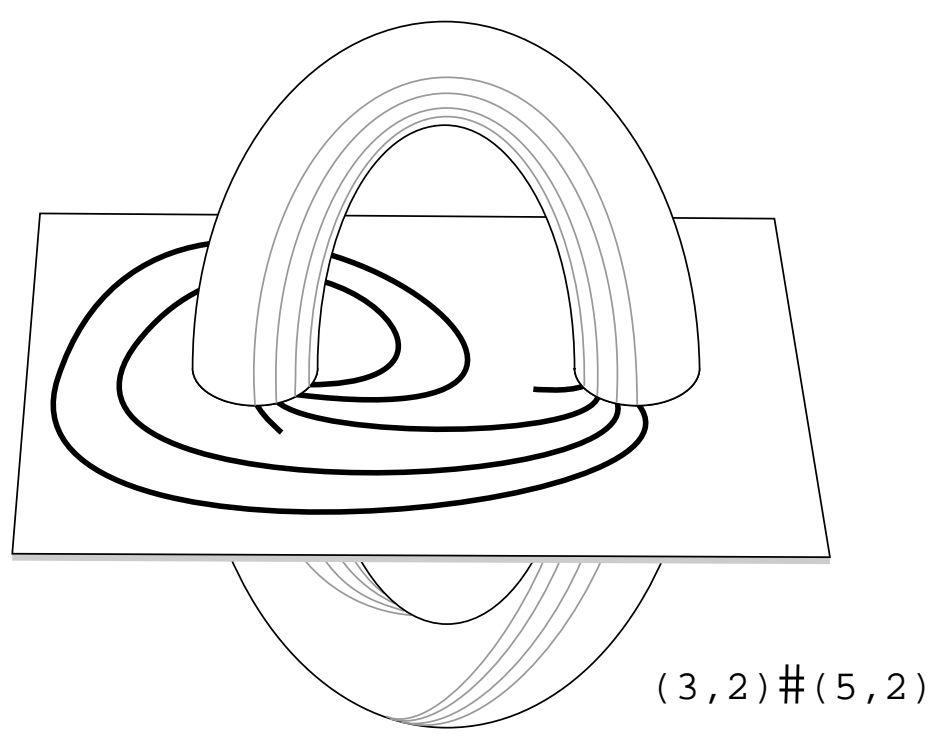

Figure 29. $A \cup \partial V$ for $(3,2) \#(5,2)$

$p>q, p=j q+a+1,0<a+1<q$ and $b=q-a-1$; if $p<q, a=q-p-1, b=p$, and $j=0$ (Figure 29). Thus for $(3,2) \#(5,-2)$, we have $j=1, a=0$, and $b=1$.

3. Teragaito's $T_{n} \# T_{-n+1}$. Although the presentation is not given here, it is clear from the construction given in $[\mathrm{MY}]$ that $|\partial A|=2$. Note that the trefoil is $T_{1}$ and the figure-eight knot is $T_{2}$.

4. Similarly, $T_{n} \#-T_{n+2}$ is a composite twisted unknot for all $n ; G_{A}$ is parametrized by the number of twists of the long arc in $G_{A}$, as illustrated in Figure 31. If $n$ is even, let $j=-(n+4) / 2$; if $n$ is odd let $j=(n+1) / 2$. 

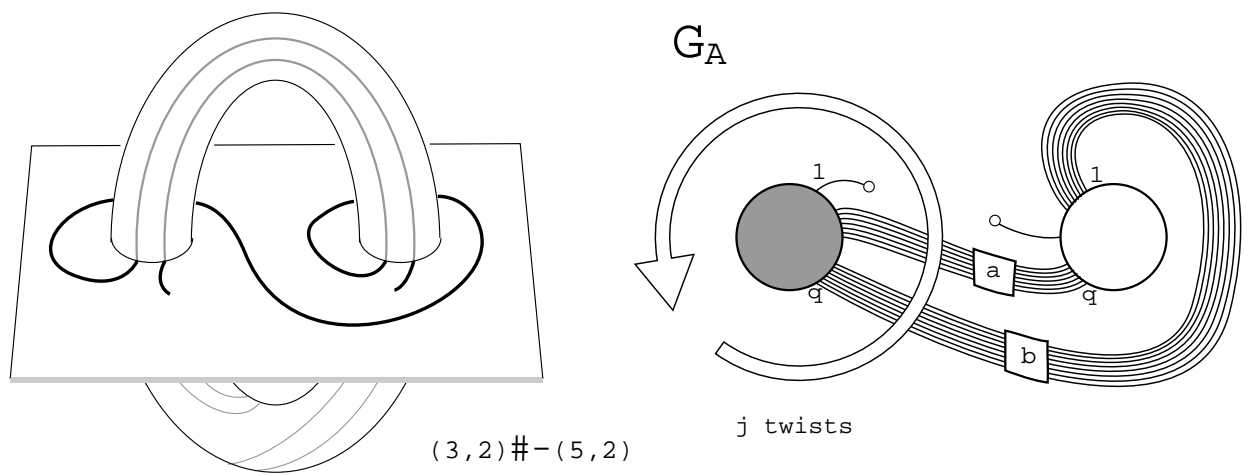

FiguRE 30. $A \cup \partial V$ for $(3,2) \#(5,-2)$ and $G_{A}$ for $(p, q) \#(p+q,-q)$
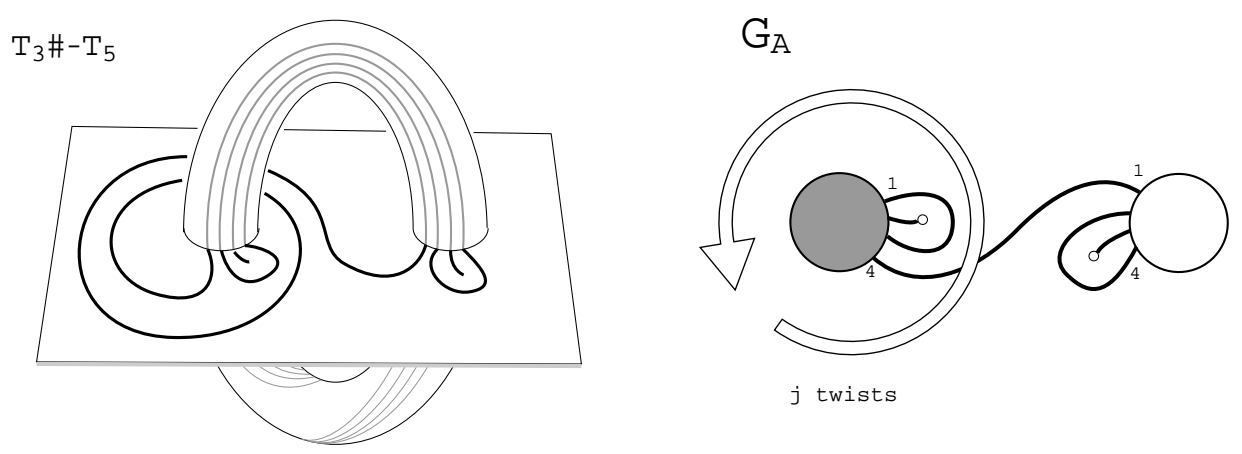

Figure $31 . T_{3} \# T_{5}$ and $G_{A}$ for $T_{n} \#-T_{n+2}$

5. For $k$ a two-bridge knot, $[\mathrm{MY}]$ points out that $k \#-k$ is a twisted unknot. The general construction in Figure 32 is to lay one bridge across a handle, and then let the other bridge follow the other arcs of the knot. Note that the cosummand pictured beneath $A$ is the reflection of the original two-bridge knot. That is, twisting the handle leaves the knot unchanged in this example.

In $[\mathrm{MY}]$, this example arose as the band sum of two unlinked unknots. By [E], if such a band sum is composite, the splitting sphere will divide the band lengthwise, and the knot will be the sum of a two-bridge knot and its reflection, exactly coinciding with this subclass of example.

6. In [T1], Teragaito gives an example of a composite twisted unknot in which one summand is an arbitrary satellite of a particular pattern, the other summand a $(p, p+1)$ torus knot.

Let $k$ be any satellite of the pattern in Figure 33. Note the equivalence of this description to viewing $k$ as any restricted band sum of two unknots linked $p+1$ times. Teragaito explicitly shows $k \#(p, p+1)$ is a composite twisted unknot.

In Figure 10, in Section 2.2, we see $A \cup \partial V$ for $k \#(3,4)$. Above $A$ the thick and thin curves are unknots linked four times, and below $A$ the thick curve describes a 


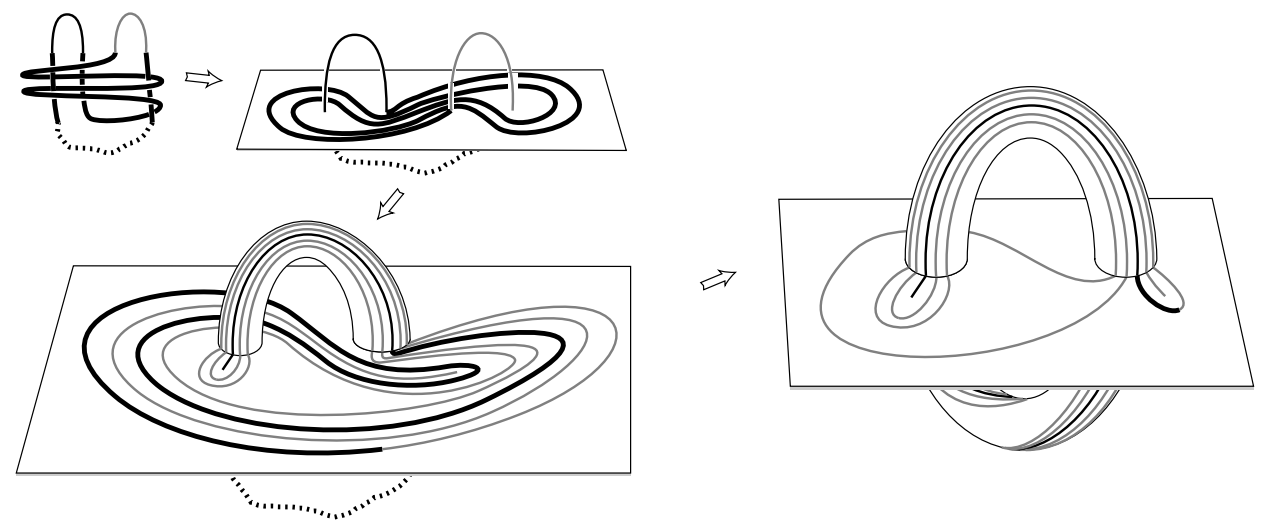

Figure 32. $A \cup \partial V$ for the sum of a two-bridge knot and its reflection

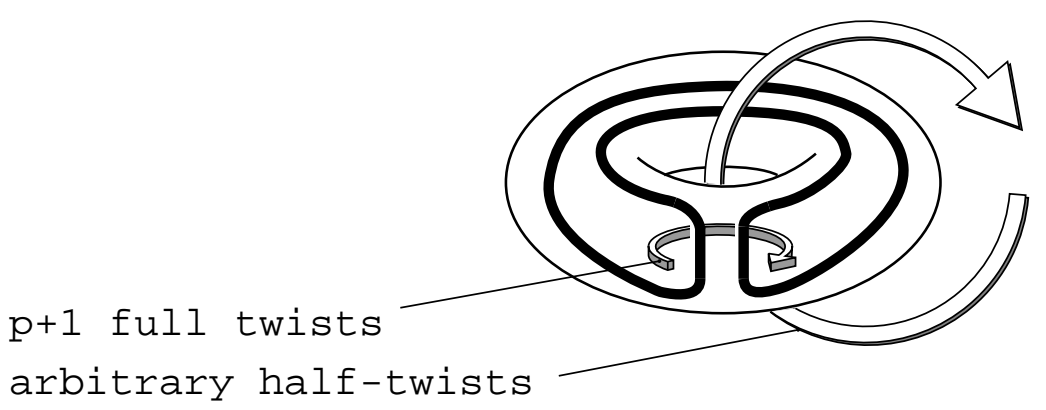

FiguRE 33. The pattern of a cosummand for $(p, p+1)$

$(3,4)$ torus knot and the thin curve bounds a disk in $V-A$. We can parametrize the graphs by $p$. There is a region of $p-1$ pairs of alternating thick and thin arcs and one of $p-2$ thin arcs, and there are other arcs as shown.

In Figure 34 is another, similar example, $k \#(4,3)$.
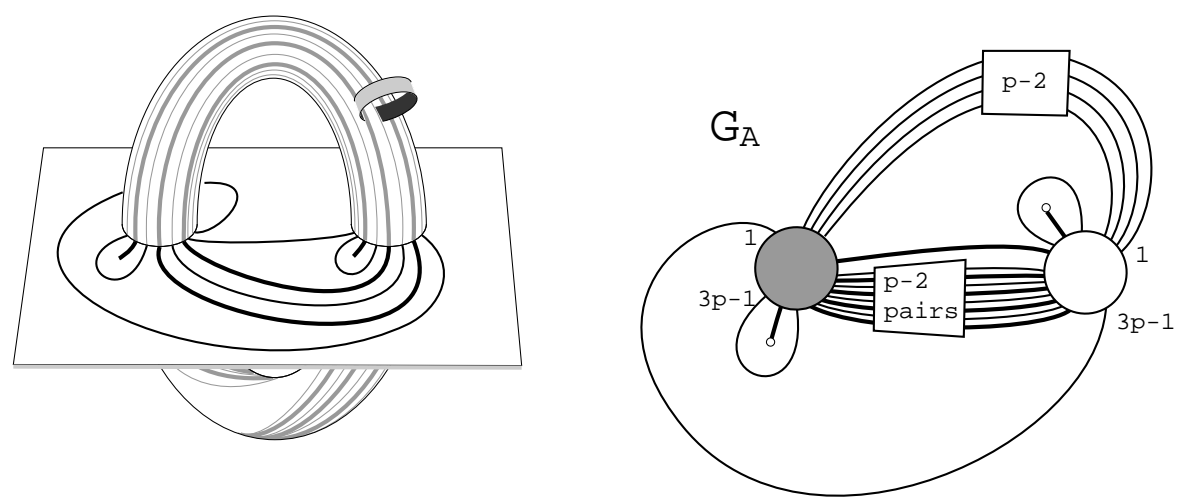

Figure 34. $A \cup \partial V$ and $G_{A}$ for another banded example 


\section{Appendix C. An infinite Class of COMPosite twisted COMPosite KNots}

Hayashi and Motegi [HMo] demonstrate that the right and left handed granny knots are related by a $(2, V)$-twisting. Above, we show this is the only pair of composite knots related by a $(2, V)$-twisting such that there is no splitting sphere missing $\partial V$, and that no such pairs can be related by such a $(3, V)$-twisting. However, every composite knot is related to an infinite collection of composite knots by $(1, V)$ twisting!

Choose any odd integer $j$. Then for any composite knot $K$ there are infinitely many $(1, V)$-twistings relating $K$ to knots of the form $K_{1} \#(j, 2)$, where $K_{1}$ is a $\left(1, V^{\prime}\right)$-twisting of $K$. The splitting sphere of $K$ meets $\partial V^{\prime}$ twice, and so $K_{1}$ is trivial exactly when the setting described in Section 2.2 holds. That is, even if $K$ is a composite twisted unknot, there are an infinite number of $\left(1, V^{\prime}\right)$-twistings that do not yield an unknot and we can see that there are indeed an infinite number of distinct non-trivial composite knots related to $K$ by a twisting.

In fact, a single infinite family of graphs gives rise to these twistings! The complex pictured has two toroidal chambers; a summand for $K$ can be placed in each with total freedom. In Figure $35, j=7$.
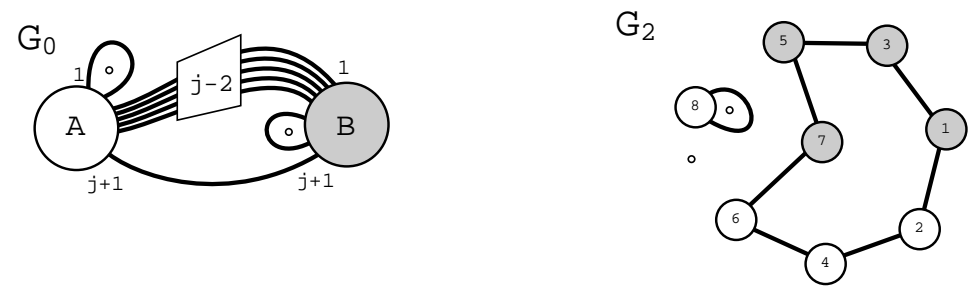

$j+1$ vertices

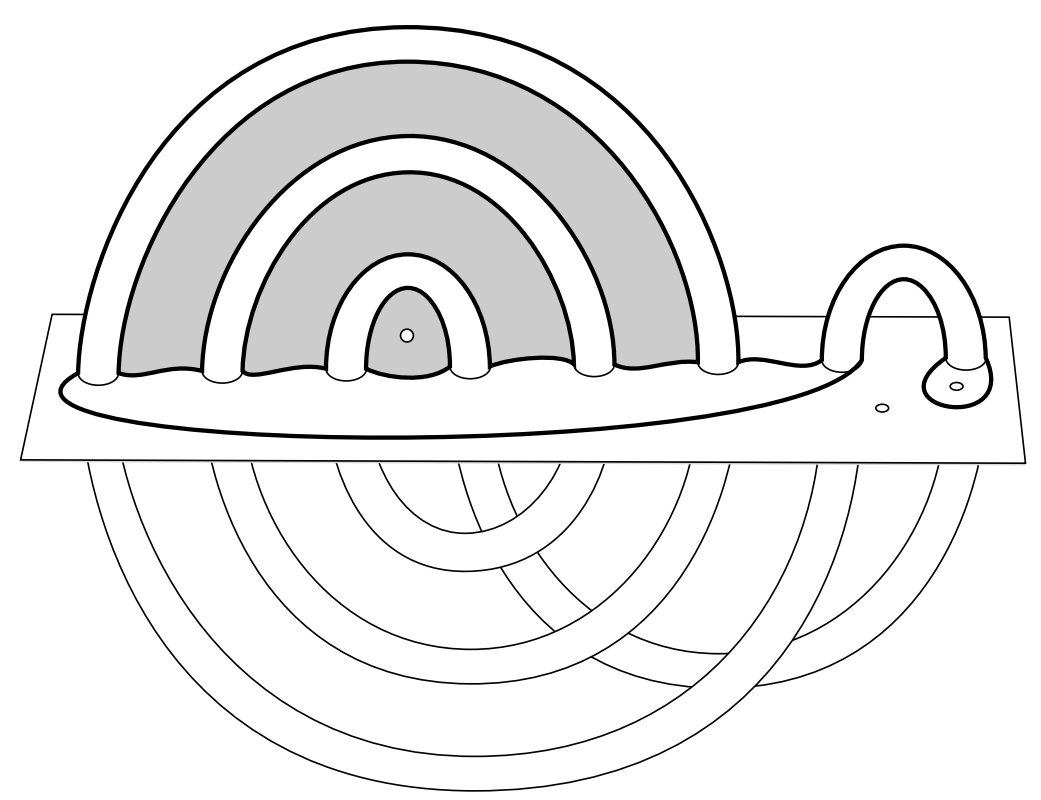

Figure 35. Graphs relating all composite $K$ to all $K_{1} \#(j, 2)$ 


\section{REFERENCES}

[CGLS] M. Culler, C. McA. Gordon, J. Luecke and P. B. Shalen, Dehn surgery on knots, Ann. of Math. (2) 125 (1987), 237-300. MR 88a:57026

[E] M. Eudave-Munoz, Band sums of links which yield composite links: The cabling conjecture for strongly invertible knots, Trans. Amer. Math. Soc. 330 (1992) no. 2, 463-501. MR 92m:57009

[GL1] C. McA. Gordon and J. Luecke, Knots are determined by their complements, J. Amer. Math. Soc. 2 (1989), 371-415. MR 90a:57006a

[GL2] C. McA. Gordon and J. Luecke, Reducible manifolds and Dehn surgery, Topology 35 (1996), 385-409. MR 97b:57013

[HMo] C. Hayashi and K. Motegi, Only single twists on unknots can produce composite knots, Trans. Amer. Math. Soc. 349 (1997), 4465-4479.

[L] J. Luecke, Notes on the knot complement problem, preprint.

[Ma] Y. Mathieu, Unknotting, knotting by twists on disks and property $(\mathrm{P})$ for knots in $S^{3}$, Knots 90, Proc. 1990 Osaka Conf. on Knot Theory and Related Topics, De Gruyter, 1992, pp. 93-102. MR 93i: 57008

[Mo] K. Motegi, Primeness of twisted knots, Proc. Amer. Math. Soc. 119 (1993), 979-983. MR 94c:57014

[MY] K. Miyazaki and A. Yasuhara, Knots that cannot be obtained from a trivial knot by twisting, preprint.

[P] W. Parry, All types implies torsion, Proc. Amer. Math. Soc. 110 (1990), 871-875. MR 91c:20079

[T1] M. Teragaito, Composite knots trivialized by twisting, Jour. Knot Theory and its Ramifications 1 (1992) 467-470. MR 93k:57023

[T2] M. Teragaito, Twisting operations and composite knots, Proc. Amer. Math. Soc. 123 (1995), 1623-1629. MR 95f:57024

Department of Mathematics, University of Arkansas, Fayetteville, Arkansas 72701

E-mail address: strauss@math.utexas.edu 